\title{
VIRGINIA TECH
}

\section{CENTER FOR \\ COMPOSITE MATERIALS \\ AND STRUCTURES}

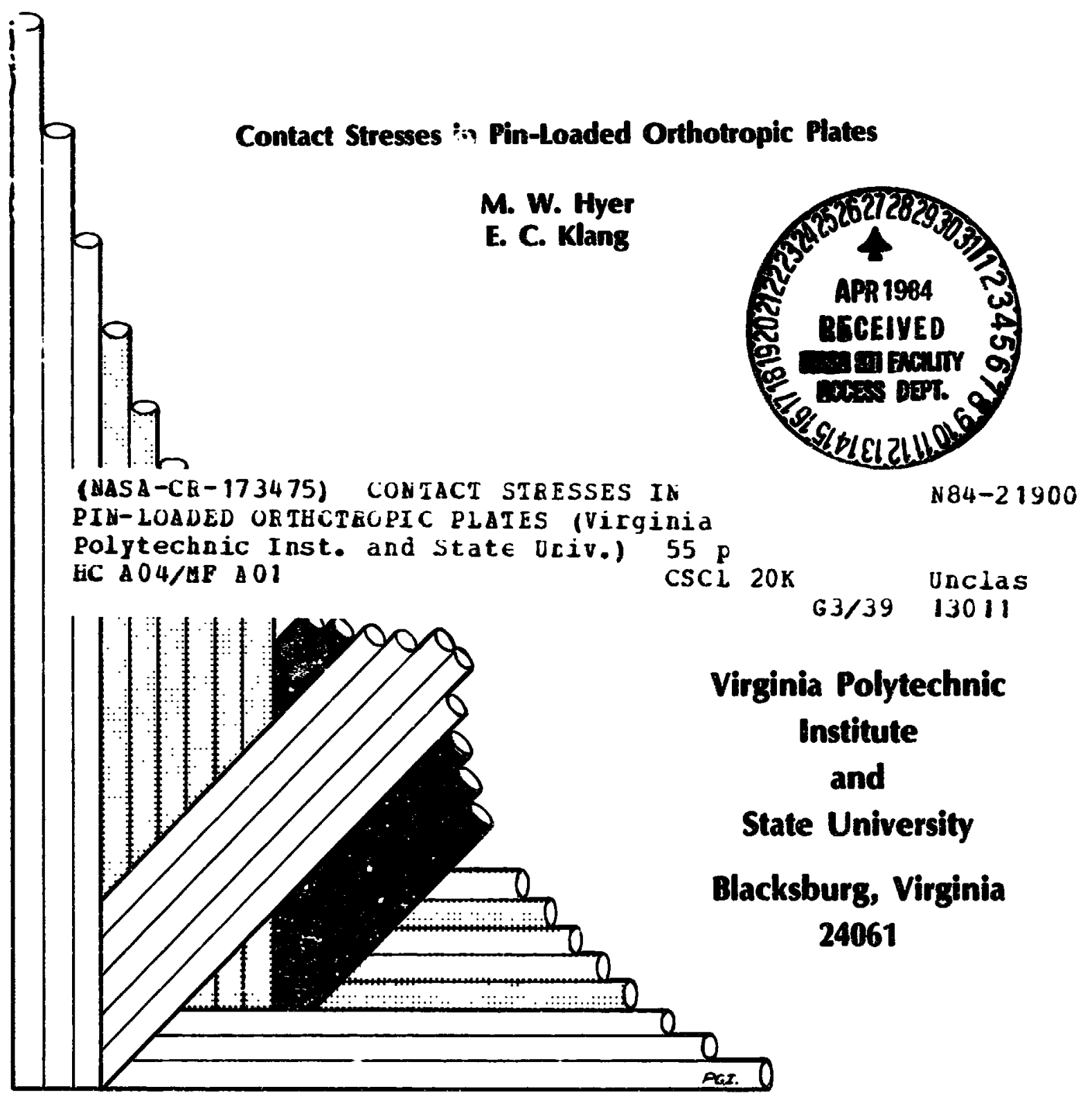


College of Engineering

Virginia Polytechnic Institute and Stite University

Blacksburg, VA 24061

VPI $-E-84-14$

Apri1, 1984

Contact Stresses in Pin-Loaded Orthotropic Plates

M. W. Hyerl

E. C. Klang 2

Department of Engineering Science \& Mechanics

Interim Report 40

The NASA-Virginia Tech Composites Program

NASA Cooperative Ayreement NAG-1-343

Prepared for: Applie.l Materials Branch

National Aeronautics \& Space Administration

Langley Research Center

Hampton, VA 23665

lProfessor, Engineering Science \& Mechanics

${ }^{2}$ Former graduate student, Engineering Science \& Mechanics, presentiy at Delft University, The Netherlands 


\section{ABSTRACT}

A study to determine the effects of pin elasticity, friction, and clearance on the stresses near the hole in a pin-loaded orthotropic plate is described. The problem is modeled as a contact elasticity problem using complex variable theory, the pin and the plate being two elastic hodies interacting through contact. This modeling is in contrast to privious works, by other investigators, which have assumed that the pin is rigid or that it exerts a known cosinusoidal radial traction on the hole boundary. Neither of these approaches explicitly involves a pin. A collocation procedure and iteration were used to obtain numerical results for a variety of plate and pin elastic properties and various levels of friction and clearance. Collocation was used to enforce the boundary conditions at a finite number of points around the hole boundary and iteration was used to find the contact and no-slip regions on the boundary. Details of the numerical scheme are discussed. The study shows that pin elasticity is not as important as clearance, friction, or the elastic properties of the plate in determining contact stresses. 


\section{Introduction}

Because of the widespread usage of fiber-reinforced composite materials in structures, there have been a number of studies to determine the stress distribution around a hole in a pin-loaded orthotropic plate [1-16]. The studies have been two dimensional plane stress analyses aimed at understanding the behavior of pinned and bolted connectors made of fiber-reinforced composite inaterials. While the plate has been modeled variocis ways, including finite-element $[1,5-8,10,12,14-16]$ and elasticity $[2-4,9,11,13]$ approaches, none of the studies have directly addressed the pin, or more importantly, pin/hole interaction. For the inost part the investiyations have assumed that either the pin was perfectly rigid $[1-9,13-15]$, or that the pin produced a known cosinusoidal radial traction on the hole boundary $[11,12,16]$. This latter assumption was first used by Bickley [17] in his study of isotropic slates. Although this has been shown to be a good approximation for isotropic plates [18], its applicability to orthotropic plates has not been checked. Neither approach requires an explicit pin model and each analysis reduces to a boundary value problem involving a single body, namely the plate. The purpose of the work reported on here was to explicitly model the pin and its interaction with the hole when determining the stresses around a hole in a pin-loaded orthotropic plate. The pin model includes pin elasticity, and friction and clearance between the pin and the hole. The problem involves two elastic bodies in contact and is much more difficult than the single body problem. One major difficulty is that the reytons of contact and no contact between the pin and hole are unknown a priori and must be solved for as part of the analysis. In addition, the presence of friction complicates the problem 
because the slip and no-slip regions must also be determined as part of the analysis. While pin elasticity $[10]$, friction $[2,3,5,7,13,14]$, and : learance $[2,3,5,15]$ have been included in previous analyses of this problem, no analysis has examined all three simultaneously. The one analysis which did include pin elasticity used finite elements to represent the pin and the plate, the pin transmitting forces to the plate through springs connecting the nodes of the pin and plate. In that study the eifects of pin alasticity were never established.

The analysis itere is based on formulating the elasticity problem in terms of conplex varias?o theory. Both the pin and the plate are linearly elastic and the plate is infinite in extent. The pin loads the plate througil a body force acting on the pin. A solution to the problem is obtained by a numerical scheme, namely a collocation procedure and iteration. The collocation procedure enforces interface and boundary conditions at the pin/hole boundary at a finite number of points. The iteration procedure is used to find the contact and no-slip regions. This paper begins by formally stating the problem. The key steps in the method of solution, which depends on having elasticity solutions for the plate and pin to sonewhat arbitrary boundary tractions, are then outlined. Following that the major components in the elasticity solutions are presented. Next the details of the collocation and iteration procedures are described. The number of collocation points, the iteration technique, the number of iterations, and solution accuracy are discussed. Finally, numerical results are presented. These results illustrate the effect of pin elasticity on the stresses around the hole and also indicate the importance of pin/ hole friction and clearance on the stress calculations. The effects of the plate's elastic properties on 
the stresses are illustrated with the computations. The paper closes with a brief discussion.

\section{Statement of the Problem}

The plate's principal material axes are aligned with an $x-y$ coordinate system. The origin of the $x-y$ system is the center of the hole, as is the origin of a cylindrical $r-9$ conrdinate system used in the analysis. The hole is of unit radius and the plate is of unit thickness. The pin loading the hole is isotropic and the net force the pin exerts on the hole is in the $x$-direction. The pin radius is $1-\lambda$ and, due to loading the hole, the center of the pin moves a distance o. Figure la depicts the geometry and coordinate systems used in the analysis. Figure $1 \mathrm{~b}$ illustrates the parameters $\lambda$ and $\delta$. The case of $\lambda$ equal to zero represents a perfectly fitting pin. As will be seen, this is a special case. A $\delta$ is in the direction of the $+x$ axis. Here $\delta$ is considered the independent loading parameter.

As the pin moves to the right, it contacts a portion of the hole. The extent of the contact region is unknown and the half contact arc is denoted as $B$. Due to friction within the contact arc there is a region of slip and a region of no slip. The half no-slip arc is denoted as $\alpha$. Outside the region of contact there is a no-contact region. Figure 2 illustrates the various regions on the pin/hole boundary. Coulomb friction is assumed to act in the contact region. The coefficient of friction is denoted as $\mu$. It is assumed a priori that there is one contact region and within that region there is one no-slip region. It is conceivable that there might be multiple regions of contact and no-contact, or slip and no-slip. However, when the stresses are actually computed 


\section{ORIGINAL PAGE IS \\ OF POOR QUALITY}

tr.: results can be examined for this possibility. If there are multiple regions of contact, tensile radial stresses will most likely develop in those regions where contact is erroneously assumed. For the cases considered there has been no evidence of this sort of behavior in the results. Also, examining the friction-induced shear stresses and their relation to the radial stresses will indicate whether multiple no-slip regions might occur. There has been no evidence of this behavior either. It is assumed that the coefficient of friction is independent of circumferential location. This assumption may not be as valid for composite materials as it would be, say, for metal.

Formaily the boundary and interface conditions between the plate and the pin can be stated as follows:

In region $I$, the no-slip region, $-\alpha<\theta<\alpha$ :

$$
\begin{aligned}
& u_{r_{\text {plate }}}=u_{r_{\text {pin }}}+\delta \cos \theta-\lambda \\
& u_{\theta \text { plate }}=u_{\theta \text { pin }}-\delta \sin \theta .
\end{aligned}
$$

In region 11 , the slip regions, $\alpha<\theta \leqslant \beta$ and $-\beta \leqslant \theta<-\alpha$ :

$$
\begin{gathered}
u_{r_{\text {plate }}}=u_{r_{\text {pin }}}+\delta \cos \theta-\lambda \\
\left|\tau_{r_{\theta}}\right|=\mu\left|\sigma_{r}\right| .
\end{gathered}
$$

In region III, the no-contact region, $\beta<\theta<2 \pi-\beta$ :

$$
\sigma_{r}=0=\tau_{r} \cdot
$$


In the above the radial and tangential elastic displacements of the pin and plate are given, respectively, by

$$
u_{r_{\text {pin }}}, u_{0 \text { pin }}, u_{r_{p l a t e}}, u_{0} \text { plate }
$$

Equations 1 and 3 express continuity of radial displacements in the contact region and eq. 2 expresses continuity of tangential displacements in the no-slip region. Equation 4 is the Coulomb friction law. Equations 5 and 6 express the traction-free conditions in the no-contact region. Because of the nature of friction, assumptions have to be made regarding the sign of $\tau_{r \theta}$. The problem can be solved assuming $\delta$ is increasing. This corresponds to a positive ${ }^{r}{ }^{\cdot}$. Alternatively, the problem can be solved assuming $\delta$ is decreasing. This corresponds to a negative 'ry. Only the former condition will be discussed here.

\section{Method of Solution}

Due to equilibrium considerations the boundary tractions acting on the pin are identical to the boundary tractions acting on the hole. As formulated hare the problem reduces to finding these boundary tractions. Once the tractions are known the stresses in the plate can be determined. Here the unknown boundary tractions are represented in the form of a complex Fourier series with unknown coefficients $A_{k}$. The series is given by

$$
(N-i T)_{\text {pin }}=\left(N-, \text { plate }=\sum_{-\infty}^{+\infty} A_{k} e^{i k \theta} \text {, all } 0\right.
$$

where, following the convention of [19], 
$N=$ the normal, or radial, traction $\sigma_{r}$ on the boundary, and
$T=$ tanjential, or shear, traction $\tau_{r \theta}$ on the boundary. Because the tangential tractions are odd functions of $\theta$ and the normal tractions are even functions of $\theta$, the $A_{k}$ are real.

Since each is a legitimate $t$ action, each term $e^{i k \theta}$ in the series produces a unique set of stresses and displacements in the plate and in the pin. In the next sections the stresses and displacements in the pin and plate due to traction $e^{i k \theta}$ will be found. If the boundary traction is $A_{k} e^{i k \theta}$, then the stresses and displacensants in the plate and pin will be weighted by $A_{k}$. If, for example, the radial displaceinent due to $N-i T=e^{i k \theta}$ is denoted as $\left(u_{r}\right)_{k}$, then the radial displacement due to traction $N-i T=A_{k} e^{i k \theta}$ is given by $A_{k}\left(u_{r}\right)_{k}$. Considering all terms in eq. 7 , the radial displacement $u_{r}$ would be the weighted sum of the effects of the individual tractions $e^{i k \theta}$. That is,

$$
u_{r}=\sum_{k=-\infty}^{\infty} A_{k}\left(u_{r}\right)_{k}
$$

If all $A_{k}$ were known, then $u_{r}$ could be calculated. The same can be said of the tangential displacement, namely

$$
u_{\theta}=\sum_{k=-\infty}^{\infty} A_{k}\left(u_{\theta}\right)_{k},
$$

where $\left(u_{\theta}\right)_{k}$ is the tangential displacement due to traction $N-i T=e^{i k \theta}$. No distinction has yet been made between the plate and the pin. The responses of the $p l a t e$ due to $N-i T=e^{i k \theta}$ are different than the responses of the pin due to $\mathrm{N}-\mathrm{iT}=\mathrm{e}^{\mathrm{ik \theta}}$. Thus the notation 


$$
\begin{aligned}
& 7 \\
& \text { ORIginal page is } \\
& \text { OF POOR QUALITY } \\
& u_{r_{\text {pin }}}=\sum_{k=-\infty}^{\infty} A_{k}\left(u_{r}\right)_{k} \text { in } \quad u_{\theta} \text { pin }=\sum_{k=-\infty}^{\infty} A_{k}\left(u_{\theta}\right)_{k} \text { pin } \\
& u_{\text {plate }}=\sum_{k=-\infty}^{\infty} A_{k}\left(u_{r}\right)_{k} \text { plate } ; u_{\theta} \text { plate }=\sum_{k=-\infty}^{\infty} A_{k}\left(u_{\theta}\right)_{k} \text { plate }
\end{aligned}
$$

distinguishes between $p$ in and plate responses. It is important to point out that since they are a result of traction $e^{i k \theta}$, the quantities

$$
\left(u_{r}\right)_{k_{\text {pin }}},\left(u_{\theta}\right)_{k_{\text {pin }}},\left(u_{r}\right)_{k} \text { plate }{ }^{\text {and }\left(u_{\theta}\right)_{k}} \text { plate }
$$

are assumed to be known. The problem will reduce to finding $A_{k}$.

To continue with the formulation of the problem, the stresses in the pin and plate are given by

$$
\left.\sigma_{\text {pin }}=\sum_{k=-\infty}^{\infty} A_{k}\left(\sigma_{i}\right)_{k} \text { in } ; \sigma_{r}\right)_{\text {ate }}=\sum_{k=-\infty}^{\infty} A_{k}\left(\sigma_{r}\right)_{k} \text { plate }
$$

$$
\begin{aligned}
& \sigma_{\theta \text { pin }}=\sum_{k=-\infty}^{\infty} A_{k}\left(\sigma_{\theta}\right)_{k \text { pin }} ; \sigma_{\theta} \text { plate }=\sum_{k=-\infty}^{\infty} A_{k}\left(\sigma_{\theta}\right)_{k} \text { plate } \\
& \tau_{r \theta_{\text {pin }}}=\sum_{k=-\infty}^{\infty} A_{k}\left(\tau_{r \theta}\right)_{k_{p i n}} ;{ }^{\tau_{r \theta}}{ } \text { plate }=\sum_{k=-\infty}^{\infty} A_{k}\left(\tau_{r \theta}\right)_{k} \text { plate }
\end{aligned}
$$

The quantities $\left(\sigma_{r}\right)_{k}, \ldots$ in $, \ldots,\left(\tau_{r \theta}\right)_{k}$ plate are the stresses in the pin and $\rho$ late due to traction $N-i T=e^{i k \theta}$. They are also considered known.

To determine the unknown coefficients $A_{k}$, a rollocation technique and iteration are used. Instead of using infinite surns in eqs. 10-19, finite suins are used. Instead of satisfying the boundary conditions 


\section{ORIGNAL PAGE IS \\ OF POOR QUALITY}

given by eqs. 1-5 at each point on the circular boundary, (i.e., at an infinite number of points) the boundary conditions are satisfied at a finite number of points. The number of terms in the truncated series is relaced to the number of collocation points on the boundary. The iterative portion of the solution involves finding the contact and no-slip arcs, $x$ and $\beta$. They, like the $A_{k}$, are unknown. However, if specific values are assumed for $\alpha$ and $\beta$, a solution to the problem with those values of $\alpha$ and $\beta$ can be found. The specific values may not yield a solution which satisfies all the boundary and interface conditions, but iteration can be used to find values which do satisfy all the conditions of the problein. The iteration procedure will be further discussed.

First, the collocation procedure is explained.

By using finite sums in eqs. 10-19 and substituting those sums into the boundary conditions for the various regions, eqs. 1-6, the method for determining the $A_{k}$ 's becones evident. Substituting the finite sums into eys. 1-6 results in:

For the no-slip region;

$$
\begin{aligned}
\sum_{k=-N}^{N} A_{k}\left(u_{r}\right)_{k} \text { plate } & =\sum_{k=-N}^{N} A_{k}\left(u_{r}\right)_{k}+\delta \cos \theta-\lambda \\
\sum_{k=-N}^{N} A_{k}\left(u_{\theta}\right)_{k} \text { plate } & =\sum_{k=-N}^{N} A_{k}\left(u_{\theta}\right)_{k}-\delta \sin \theta .
\end{aligned}
$$

For the slip regions;

$$
\begin{gathered}
\sum_{k=-N=N}^{N} A_{k}\left(u_{r}\right)_{k} \text { plate }=\sum_{k=-N}^{N} A_{k}\left(u_{r}\right)_{k}+\delta \cos \theta-\lambda \\
\sum_{k=-N}^{N} A_{k}\left(\left(\tau_{r \theta}\right)_{k}-\mu\left(\sigma_{r}\right)_{k}\right)=0 .
\end{gathered}
$$


For the no contact region;

$$
\begin{aligned}
& \sum_{k=-N}^{N} A_{k}\left(\sigma_{r}\right)_{k}=0, \\
& \sum_{k=-N}^{N} A_{k}\left(\tau_{r \theta}\right)_{k}=r .
\end{aligned}
$$

There are $2 \mathrm{~N}+1$ terins in each sum. If the boundary conditions are satisfied at $2 N+1$ discrete points around the circular boundary, a set of $2 N+1$ linear algebraic equations can be for.ned from which to solve for the $A_{k}$. Since the proper symnetry conditions have been incorporated into the solution, the $2 N+1$ discrete points need only be distributed over onehalf the hole circumference, $0 \leqslant \theta \leqslant \pi$. The assuined values of $\alpha$ and $\beta$ determine over what range of $\theta$ eqs. 20 and 21 are enforced, over what other range eys. 22 and 23 are enforced, and over what range eqs. 2.4 and 25 are enforced. Since the tractions on the plate and pin are identical, neither the plate nor the $\mu$ in is called out specifically in eys. 24 and 25. Though it is not specifically noted, attention must be given to signs in ey. 23. In addition, strictly speaking, by eq. 7

and

$$
\left.\begin{array}{l}
\left(\sigma_{r}\right)_{\text {pin }}=\left(\sigma_{r}\right)_{k \text { plate }}=\cos (k \theta) \\
\left(\tau_{r \theta}\right)_{k \text { pin }}=\left(\tau_{r \theta}\right)_{k \text { plate }}=-\sin (k \theta) .
\end{array}\right\} \begin{aligned}
& \text { at the } \\
& \text { boundary }
\end{aligned}
$$

Equations 23-25 thus can be written as 


$$
\begin{gathered}
\sum_{k=-N}^{N} A_{k}(\sin (k \theta)+\mu \cos (k \theta))=0 . \\
\sum_{k=-N}^{N} A_{k} \cos (k \theta)=0 \\
\sum_{k=-N}^{N} A_{k} \sin (k \theta)=0 .
\end{gathered}
$$

Expanding eys. 20-22 and 23a-25a, evaluating them at $2 \mathrm{~N}+\mathrm{i}$ circumferential $(\theta)$ locations, rearranging slightly, and putting thern into matrix notation ieads to equations of the form

$$
[C]\{A\}=\{B\}
$$

Expanding, these equations can be written as 


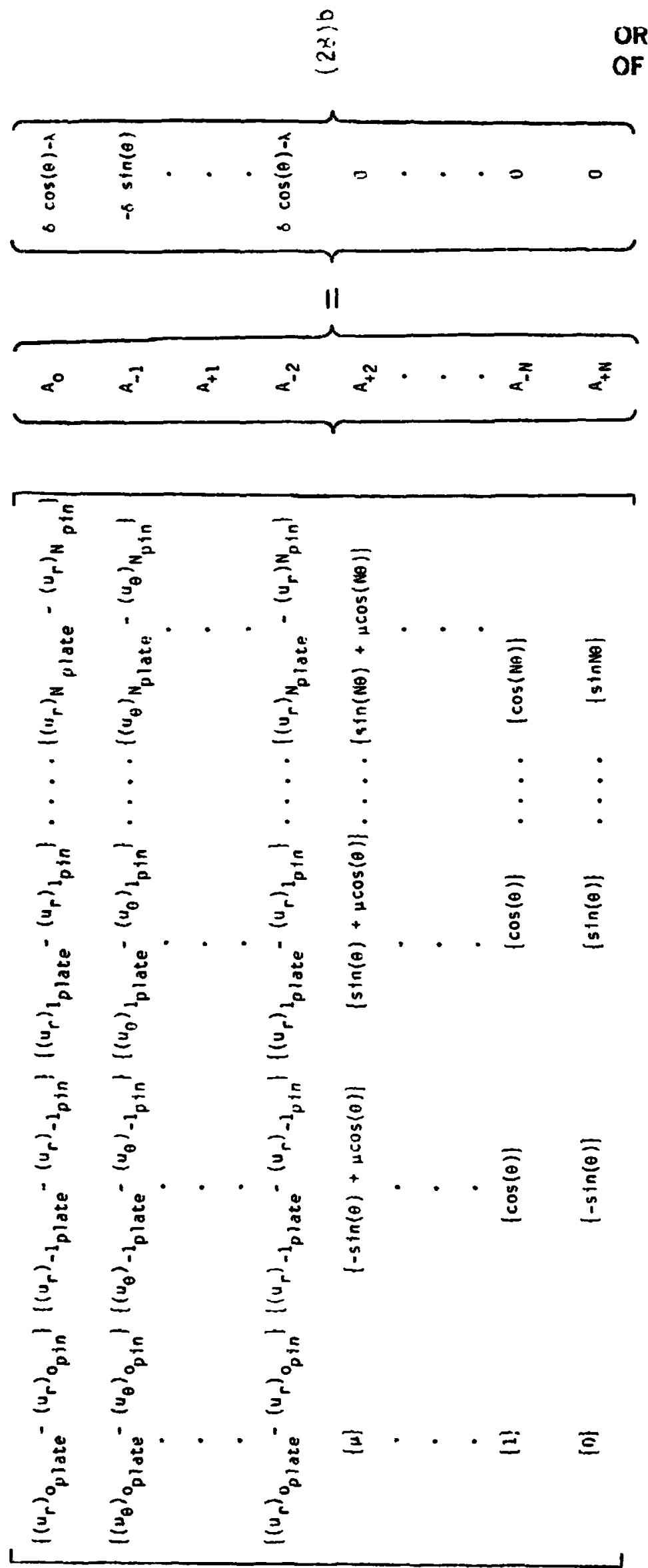

ORININAL PAEE IS

OF POOR QUALITY 
The torms in the top two lines in the cuefficient matrix [C] and in the vector (3) represent terms evaluated at various $\theta$ locations in the no-slip zone, the terms in the second two lines represent terms evaluated at various $\theta$ locations in the slip zone, and the last two lines represunt :erms evaluated at varioss $\theta$ locations in the no-contact zone. It is important to point out that with a perfectly fitting pin $\lambda=0$ and each element on the rignt-hand-side of eq. 28 changes, for example, by a factur of two when $\delta$ is doubled. Each $A_{k}$ simply doubles and the spatial variatio of $N-i T$, with $\theta$, remains unchanged. Thus $\alpha$ and $\beta$ are unafferted by s.. With $\lambda \neq 0$, doubling is does not result in a doubling of the right-hand-sije of eq. 28. Each $A_{k}$ changes by a different amount and thus the spatial variation of $\mathrm{N}-\mathrm{iT}$ varies. This transiates into $S$ directly affecting $\alpha$ and $\beta$. The perfect fit case is therefore quite special and is often referred to as the linear case.

To implement the solution procedure the responses of the plate and pin to boundary traction $e^{i k \theta}$, namely $\left(u_{r}\right)_{k}, u_{\text {in }},\left(u_{r}\right)_{k}, l_{a t e}, \ldots$, must be found. This follows.

Response of Pin to $e^{i k \theta}$

In reality, the pin transmitts a load to the hole boundary because it is loaded by some external mechanism. Commorily the $p$ in is in double shear, being loaded by two other plates parallel to and on either side of the plate under discussion. This is shown in fig. 3a. To be sure, there are bending stresses in the $p$ in and the exact determination of stresses in the pin requires a three-dimensional analysis. However, for the seyment of pin within the thickness of the plate the situation shown in fig. $3 b$ is quite accurate. This segment of the pin, shown as a disk, 
is loaded by shear stresses from portions of the pin on eitner side of the plate, and by the contact stresses. Compared to the length of the pin, in region of the $p$ in within the thickness of the plate is small. However, the fact that the pin actualiy iis lonoth does influence the deformation of the pin within the thickness region. Here the pin is assumed to be in a state of plane deformation and the shear stresses in fig. 3b are assumed to be spatially uniform over the pin cross-sectional drea. Furthermore, for purposes of analysis, the effects of the uniform shear traction are assumed to be equivalent to a uniformly distributed body force acting in the $x$ direction within the pin. An alternative pin model would be to assume the disk representing the pin is loaded at its cenier by a concentrated force. This model was not considered because it was felt to be too harsh an idealization of the forces actually transmitted to the portion of the pin within the thickness of the plate. A concentrated force model was adapted by Rao [20] in a study of isotropic plates.

With the model adopted here, the equilibrium equations for the pin are

$\frac{\partial \sigma_{r}}{\partial r}+\frac{1}{r} \frac{\partial \tau_{r \theta}}{\partial \theta}+\frac{\sigma_{r}-\sigma_{\theta}}{r}+b_{r}=0, \frac{1}{r} \frac{\partial \sigma_{\theta}}{\partial \theta}+\frac{\partial \tau_{r \theta}}{\partial r}+\frac{2 \tau_{r \theta}}{r}+b_{\theta}=0$, where $b_{r}$ and $b_{\theta}$ are the components of the body force. Milne-Thomp son [21] discusses the complex variable approach for the case of a body force and so only the primary features of the analysis are presented nere.

To effectively use complex variables, it is convenient to express the body force components in terms of a scalar potential, $V$, i.e.

$$
b_{r}=-\frac{\partial V(r, \theta)}{\partial r} \text { and } b_{\theta}=-\frac{1}{r} \frac{\partial V(r, \theta)}{\partial \theta} \text {. }
$$


Defining a stress function $F(r, \theta)$ as

$$
\begin{aligned}
& \sigma_{r}=\frac{1}{r} \frac{\partial F}{\partial r}+\frac{1}{r^{2}} \frac{\partial^{2} F}{\partial \theta^{2}}+V ; \sigma_{\theta}=\frac{\partial^{2} F}{\partial r^{2}}+V ; \quad \text { (31)a,b } \\
& \tau_{r \theta}=\frac{1}{r^{2}} \frac{\partial^{2} F}{\partial \theta^{2}}-\frac{1}{r} \frac{\partial^{2} F}{\partial r \partial \theta}
\end{aligned}
$$

automatically satisfies the equilibrium equations. 'Jsing Hooke's Law for an isotropic material, with Poisson's ratio $v$, and substituting the strains in terms of the stresses into the only nontrivial compatibility equation leads to the following equation for $F$ and $V$ :

$$
\nabla^{4} F+\kappa \nabla^{2} V=0
$$

where

$$
\kappa=(1-2 v) /(1-v)
$$

Defininy

$$
v=\nabla^{2} Q
$$

where $P$ is anvther scalar function, eq. 32 becomes

$$
\nabla^{4}(F+K Q)=0
$$

Defininy

$$
z=x+i y=r e^{i \theta}
$$

the solution for $F$ can be expressed in terms of two complex functions, $\phi$ and $\chi$, and $Q$ as 


$$
F=\frac{1}{2}\left[\bar{z}_{\phi}(z)+z \bar{\phi}(\bar{z})+x(z)+\bar{x}(\bar{z})\right]-k Q(z, \bar{z}) .
$$

The first and second fundamental stress combinations are written as

$$
\begin{gathered}
\sigma_{\theta}+\sigma_{r}=2(\bar{q}(z)+\bar{\Phi}(\bar{z}))+(8-4 k) \frac{\partial^{2} Q(z, \bar{z})}{\partial z \partial \bar{z}}, \\
\sigma_{g}-\sigma_{r}+i 2 \tau_{r \theta}=2\left(\bar{z} \Phi^{\prime}(z)+\Psi(z)-2 k \frac{\partial^{2} Q(z, \bar{z})}{\partial z^{2}}\right) e^{2 i \theta},
\end{gathered}
$$

and the eiastic displacements are yiven as

$$
\left.\left(u_{r}+i \nu_{\theta}\right)=\frac{1}{2 G}((3-4 v)) \phi(z)-2 \bar{q}^{\prime}(\bar{z})-\bar{\psi}(\bar{z})+2 \kappa \frac{\partial Q(z, \bar{z})}{\partial \bar{z}}\right) e^{-i \theta} .
$$

In the above the prime, ', denotes differentiation with respect to the argument, $G$ is the material's shear modulus, and

$$
\left.\Phi^{\prime} z\right)=\phi^{\prime}(z), \varphi(z)=\psi^{\prime}(z), \text { and } \phi(z)=\chi^{\prime}(z) \text {. }
$$

The overbar, ${ }^{-}$, denotes comp lex conjugate.

At the boundary

$$
\sigma_{r}-i_{\tau}{ }_{r \theta}=N-i T=\sum_{k=-\infty}^{\infty} A_{k} e^{i k \theta} .
$$

Therefore the stress co'm ination most useful for matching traction boundary conditio is obtained hy subtracting eq. 39 from eq. 38. The result is

$$
\begin{gathered}
o_{r}-i \tau_{r \theta}=\phi(z)+\bar{\Phi}(\bar{z})-\left(\bar{z} \Phi^{\prime}(z)+\Psi(z)\right) \mathrm{e}^{2 i \theta} \\
+i(2-\kappa) \frac{\partial^{2} u(z, \bar{z})}{\partial \bar{z}_{\partial j} z}+2 k \frac{\partial^{2} \Omega(z, \bar{z})}{\partial^{2} z} \mathrm{e}^{i 2 \theta}
\end{gathered}
$$


Since the pin domain is simply connected, $\phi(z)$ and $\psi(z)$ can be representen by

$$
\begin{aligned}
& \varphi(z)=\sum_{k=0}^{\infty} a_{k} z^{k}=\sum_{k=0}^{\infty} a_{k} r^{k} e^{i k \theta} \\
& \Psi(z)=\sum_{k=0}^{\infty} b_{k} z^{k}=\sum_{k=0}^{\infty} b_{k} r^{k} e^{i k \theta},
\end{aligned}
$$

$a_{k}$ and $b_{k}$ being constants, possibly complex.

To complete the pin analysis, 0 needs to be determined. MilneThompson [21] presents a derivation of $Q$ for a more general body force and those results can be reduced to the situation here. The result is

$$
Q=-\frac{{ }^{b} x}{16}\left(z^{2} \bar{z}+(\bar{z})^{2} z\right)
$$

where $b_{x}$ is the body force in the $x$ direction, $b_{x}$ being given by

$$
b_{x}=\sqrt{b_{r}^{2}+b_{\theta}^{2}}
$$

Substituting eqs. 44 and 45 into eqs. $38-40$, the stresses and displacements can be determined in terms of $a_{k}, b_{k}$ and $b_{x}$. Specifically the stress combination of eq. 43 evaluated on the boundary is

$$
\begin{aligned}
& \sum_{k=-\infty}^{\infty} A_{k} e^{i k \theta}=\sum_{k=0}^{\infty} a_{k} R^{k} e^{i k \theta}+\sum_{k=0}^{\infty} a_{k} R^{k} e^{-i k i)} \\
& -\sum_{k=1}^{\infty} k a_{k} R^{k} e^{i k \theta}-\sum_{k=2}^{\infty} b_{k-2^{R}} R^{k-2} e^{i k j} \\
& -\frac{b_{x} R(2-k)}{4}\left(e^{i \theta}+e^{-i \theta}\right)-\frac{b_{x} R_{k}}{4} e^{i \theta}
\end{aligned}
$$


Here $R$ is the radius of the $p$ in. Since the hole in the plate is of unit radius and the clearance between the pin and hole is $\lambda$,

$$
R=1-\lambda
$$

Using eq. 47 and setting dll $A_{k}$ 's to zero except one, and setting that $A_{k}$ equal to one, constants $a_{k}$ and $b_{k}$ can be found by matching terms on the left and right sides of eq. 47. The solution of the pin to traction $N-i T=e^{i k \theta}$ is now available. Table 1 shows the result of matching terms. The values of $a_{k}$ and $b_{k}$ from Table 1 can be substituted into eq. 44 and those results into eq. 40 to find explicit expressions for $\left(u_{r}\right)_{k_{p i n}}$ and $\left(u_{\theta}\right)_{k_{p i n}}$. These expressions, in turn, are used in eq. 28. It should be mentioned that $b_{x}$ is not an independent constant. The body force and the boundary tractions must be in equilibrium. Except for $k=+1$, boundary tractions of the form $e^{i k \theta}$ dc not iroduce a net force on the boundary. Therefore, $b_{x}$ is identically zero except for the case when $A_{1}$ is nonzero. For the boundary traction $A_{1} e^{i \theta}$,

$$
b_{x}=\frac{2 A_{1}}{R}
$$

It should also be mentioned that in determining the results of Table 1 , the imaginary part of a was arbitrarily assumed to be zero.

Response of Plate to $\mathrm{e}^{i k \theta}$

The plate is assumed to be in a state of plane stress with tractions of the form

$$
N-i T=\sum_{k=-\infty}^{\infty} A_{k} e^{i k \theta}
$$




\section{ORIGANAL PAGE is}

OF POOR QUALITY

\section{Table 1}

Coefficients of the stress functions $\Phi$ and $\psi$ for $p i n$.

\begin{tabular}{|c|c|c|}
\hline $\begin{array}{c}\text { nonzero } \\
A_{k}\end{array}$ & $\begin{array}{c}\text { nonzero } \\
\text { a's }\end{array}$ & $\begin{array}{c}\text { nonzero } \\
\text { b's }\end{array}$ \\
\hline$A_{3}=1$ & $a_{0}=1$ & $a l l b^{\prime} s=0$ \\
\hline$A_{-1}=1$ & $a_{1}=\frac{1}{R}$ & $a l l b^{\prime} s=0$ \\
\hline$A_{1}=1$ & $a_{1}=\frac{k-2}{2 R}$ & $a_{11} b^{\prime} s=0$ \\
\hline$A_{-2}=1$ & $a_{2}=\frac{1}{R^{2}}$ & $b_{0}=-1$ \\
\hline$A_{2}=1$ & $a l l a^{\prime} s=0$ & $b_{0}=-1$ \\
\hline$A_{-3}=1$ & $a_{3}=\frac{1}{R^{3}}$ & $b_{1}=-\frac{2}{R}$ \\
\hline$A_{3}=1$ & all $a^{\prime \prime} s=0$ & $b_{1}=-\frac{1}{R}$ \\
\hline - & • & - \\
\hline$:$ & • & • \\
\hline$A_{-k}=1$ & $a_{k}=\frac{1}{R^{k}}$ & $b_{k-2}=\frac{-(k-1)}{R^{k-2}}$ \\
\hline$A_{+k}=1$ & all $a^{\prime} s=0$ & $b_{k-2}=\frac{-1}{k^{k-2}}$ \\
\hline
\end{tabular}


acting on the hole boundary. These surface tractions are in equilibrium with vanishingly small stresses at infinity. The plate's principal material axes are aligned with the $x$ and $y$ axes and so the constitutive Dehavior can be written as

$$
\left\{\begin{array}{c}
\varepsilon_{x} \\
\varepsilon_{y} \\
r_{x y}
\end{array}\right\}=\left[\begin{array}{ccc}
\frac{1}{E_{x}} & -\frac{v_{x y}}{E_{x}} & 0 \\
-\frac{v_{x y}}{E_{x}} & \frac{1}{E_{y}} & 0 \\
0 & 0 & \frac{1}{G_{x y}}
\end{array}\right]\left\{\begin{array}{c}
\sigma_{x} \\
\sigma_{y} \\
\tau_{x y}
\end{array}\right\} .
$$

Here $E_{x}$ and $E_{y}$ are Young's moduli in the $x$ and $y$ directions, respectively, $v_{x y}$ is the Poisson's ratio relating contraction in the $y$ direction to the extension in the $x$ direction caused by a tensile stress in the $x$ direction, and $G_{x y}$ is the shear modulus in the $x-y$ plane. The equilibrium equations in the principal material system are

$$
\frac{\partial \sigma_{x}}{\partial x}+\frac{\partial \tau_{x y}}{\partial y}=0 ; \quad \frac{\partial \tau_{x y}}{\partial x}+\frac{\partial \sigma_{y}}{\partial y}=0 .
$$

Defining a stress function as

$$
\sigma_{x}=\frac{\partial^{2} F}{\partial y^{2}} ; \quad \sigma_{y}=\frac{\partial^{2} F}{\partial x^{2}} ; \quad \tau_{x y}=-\frac{\partial^{2} F}{\partial x \partial y}
$$

the equilibrium equations are satisfied. Using the constititive equations and the stress function, the only nontrivial compatibility equation can be used to determine $F$. This equation is

$$
\frac{\partial^{4} F}{\partial y^{4}}+\left(\frac{E_{x}}{G_{x y}}-2 v_{x y}\right) \frac{\partial^{4} F}{\partial x^{2} \partial y^{2}}+\frac{E_{x}}{E_{y}} \frac{g^{\prime}: F}{\partial x^{4}}=0 .
$$




\section{ORIGINAL PAGE IS \\ OF POOR QUALITY}

Lekhnitskii [22] and Milne-Thompson [21] discuss the general solution to this equation and associated boundary conditions, while deJong $[4,9,13]$, Oplinger and Gandhi [2,3], and Mangalgiri [7] discuss the solution in the context of a plate with a hole. Therefore, details of the solution are omitted here.

The solution to eq. 53 has the form

$$
F=\frac{1}{4}\left[W_{1}\left(z_{1}\right)+W_{2}\left(z_{2}\right)+\bar{W}_{1}\left(\bar{z}_{1}\right)+\bar{W}_{2}\left(\bar{z}_{2}\right)\right] \text {, }
$$

where

$$
z_{1}=x+\mu_{1} y \text { and } z_{2}=x+\mu_{2} y
$$

and $\mu_{1}$ and $\mu_{2}$ are two roots of the characteristic equation associated with eq. 53, namely

$$
\mu^{4}+\left(\frac{E_{x}}{G_{x y}}-2 v_{x y}\right) \mu^{2}+\frac{E_{x}}{E_{y}}=0 .
$$

(There are four roots to this equation but they occur in complex conjugate pairs, i.e. $\bar{\mu}_{3}=\mu_{1}$ and $\bar{\mu}_{4}=\mu_{2}$ ). The functions $W_{1}$ and $W_{2}$ are tobe-determined functions of the two transformed variables $z_{1}$ and $z_{2}$. With the definitions of eq. 55

$$
z_{k}=r_{k} z+\delta_{k} \bar{z}, k=1,2 \text {, }
$$

where

$$
\gamma_{k}=\frac{1-i_{\mu_{k}}}{2} \text { and } \delta_{k}=\frac{1+i_{\mu_{k}}}{2} \text {. }
$$

The first and second fundamental stress combinations are 


$$
\begin{aligned}
& 21
\end{aligned}
$$

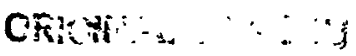

$$
\begin{aligned}
& \sigma_{r}+\sigma_{\theta}=\gamma_{1} \delta_{1} W_{1}^{\prime \prime}\left(z_{1}\right)+\gamma_{2} \delta_{2} W_{2}^{\prime \prime}\left(z_{2}\right)+\bar{\gamma}_{1} \bar{\delta}_{1} \bar{W}_{1}^{\prime \prime}\left(\bar{z}_{1}\right)+ \\
& \bar{\gamma}_{2} \bar{\delta}_{2} \bar{W}_{2}^{\prime \prime}\left(\bar{z}_{2}\right)
\end{aligned}
$$$$
\text { of Foun }
$$

and

$$
\begin{aligned}
\sigma_{\theta}-\sigma_{r}+2 i \tau_{r \theta} & =\left(\gamma_{1}^{2} W_{1}^{\prime \prime}\left(z_{1}\right)+\gamma_{2}^{2} w_{2}^{\prime \prime}\left(z_{2}\right)+\bar{\delta}_{1}^{2} \bar{w}_{1}^{\prime \prime}\left(\bar{z}_{1}\right)\right. \\
& \left.+\bar{\delta}_{2}^{2} \bar{w}_{2}^{\prime \prime}\left(\bar{z}_{2}\right)\right) e^{i 2 \theta} .
\end{aligned}
$$

The displacements are

$$
\left(u_{r}+i u_{\theta}\right)=\frac{1}{4}\left[p_{1} w_{1}^{\prime}\left(z_{1}\right)+p_{2} w_{2}^{\prime}\left(z_{2}\right)+\bar{z}_{1} \bar{w}_{1}^{\prime}\left(\bar{z}_{1}\right)+\bar{q}_{2} \bar{w}_{2}^{\prime}\left(\bar{z}_{2}\right)\right] e^{-i \theta},
$$

with

$$
\begin{aligned}
& p_{1}=-\frac{\nu_{x y}}{E_{x}}+\frac{\mu_{1}^{2}}{E_{x}}+\frac{i}{\mu_{1}}\left(\frac{1}{E_{y}}-\frac{\nu_{x y}}{E_{x}} \mu_{1}^{2}\right) \\
& p_{2}=-\frac{\nu_{x y}}{E_{x}}+\frac{\mu_{2}^{2}}{E_{x}}+\frac{i}{\mu_{2}}-\left(\frac{1}{E_{y}}-\frac{v_{x y}}{E_{x}} \mu_{2}^{2}\right) \\
& \bar{q}_{1}=-\frac{\nu_{x y}}{E_{x}}+\frac{\mu_{1}^{2}}{E_{x}}+\frac{i}{\mu_{1}}\left(\frac{1}{E_{y}}-\frac{\nu_{x y}}{E_{x}} \bar{\mu}_{1}^{2}\right) \\
& \bar{q}_{2}=-\frac{\nu_{x y}}{E_{x}}+\frac{\bar{\mu}_{2}^{2}}{E_{x}}+\frac{i}{\bar{\mu}_{2}}\left(\frac{1}{E_{y}}-\frac{\nu_{x y}}{E_{y}} \mu_{2}^{2}\right) .
\end{aligned}
$$

The stress combination $\sigma_{r}-i \tau_{r}$ can be formed, as was done with the pin, to match the traction N - iT at the boundary. Howevor, the definitions of eq. 55 transform the problem from the $z$ plane to the $z_{1}$ and $z_{2}$ planes. These transiormations distnrt the unit circle (hole) of the $z$ plane into ellipses in the $z_{1}$ and $z_{2}$ planes. The boundary conditions are not defined in these planes. A second set of transformations which map the $z_{1}$ and $z_{2}$ elliptical boundaries into unit circles is 
necessary. These transformations map the $z_{1}$ plane onto the $\zeta_{1} p l$ ane and the $z_{2}$ plane onto the $\zeta_{2}$ plane. These transformations are

$$
\begin{aligned}
& r_{2}=\frac{z_{1}+\sqrt{z_{1}^{2}-4 \gamma_{1} \delta_{1}}}{\frac{2 \gamma_{1}}{z_{2}-4 \gamma_{2} \delta_{2}}} \\
& r_{2}=\frac{z_{2}+\sqrt{2 \gamma_{2}} .}{} .
\end{aligned}
$$

As can be seen, when $z=e^{i \theta}, \zeta_{1}=\zeta_{2}=e^{i \theta}$, thus preserving the shape of the unit boundary. The inverse transformations are

$$
\begin{aligned}
& z_{1}=m_{1}\left(\zeta_{1}\right)=\gamma_{1} \zeta_{1}+\frac{\delta_{1}}{\zeta_{1}} \\
& z_{2}=m_{2}\left(\zeta_{2}\right)=\gamma_{2} \zeta_{2}+\frac{\delta_{2}}{\zeta_{2}} .
\end{aligned}
$$

With the change of variables, new nomenclature can be introduced, nannely,

$$
W_{k}^{\prime}\left(z_{k}\right)=W_{k}^{\prime}\left(z_{k}\left(\zeta_{k}\right)\right) \equiv 2 \Phi_{k}\left(\zeta_{k}\right), \quad k=1,2 .
$$

With this

$$
W_{k}^{\prime \prime}\left(z_{k}\right)=2 \phi_{k}^{\prime}\left(\zeta_{k}\right) \frac{d \zeta_{k}}{d z_{k}}, \quad k=1,2 \text {. }
$$

The functions $\phi_{k}\left(z_{k}\right)$ are of the form

$$
\begin{aligned}
& \phi_{1}\left(\zeta_{1}\right)=a_{0} \ln \zeta_{1}+\sum_{k=1}^{\infty} \frac{a_{k}}{\zeta_{1}^{k}} \\
& \phi_{2}\left(\zeta_{2}\right)=b_{0} \ln \zeta_{2}+\sum_{!:=2}^{\infty} \frac{b_{k}}{\zeta_{2}^{k}},
\end{aligned}
$$




\section{ORIGINAL PAGE IS'
OF POOR QUALITY}

$\therefore$ here the $a_{k}$ 's and $b_{k}$ 's are constants. The logrithmic terms ref lect the fact that there is a net force on the boundary of the singly connected plate.

For the plate the analog to eq. 43 , evaluated on the hole boundary, is

$\left.\left(\sigma_{r}-i \tau_{r \theta}\right)\right|_{z=e^{i \theta}}=\left.\left(\bar{\delta}_{1} \bar{\phi}_{1}^{\prime}\left(\zeta_{1}\right)+\bar{\delta}_{2} \bar{\phi}_{2}^{\prime}\left(\bar{\zeta}_{2}\right)-\left(\gamma_{1} \phi_{1}^{\prime}\left(\zeta_{1}\right)+\gamma_{2} \phi_{2}^{\prime}\left(\zeta_{2}\right)\right) e^{i 2 \theta}\right)\right|_{z=e^{i \theta}}$

Using eqs. 42 and 67 , eq. 68 becoines

$$
\begin{gathered}
\sum_{k=-\infty}^{\infty} A_{k} e^{i k \theta}=\bar{\delta}_{1}\left(\bar{a}_{0} e^{i \theta}-\sum_{k=1}^{\infty} k \bar{a}_{k} e^{i(k+1) \theta}\right)+ \\
\bar{\delta}_{2}\left(\bar{b}_{0} e^{i \theta}-\sum_{k=1}^{\infty} k \bar{b}_{k} e^{i(k+1) \theta}\right)-\gamma_{1}\left(a_{0} e^{i \theta}-\sum_{k=1}^{\infty} k a_{k} e^{-i(k-1) \theta}\right) \\
-r_{2}\left(b_{0} e^{i \theta}-\sum_{k=1}^{\infty} k b_{k} e^{-i(k-1) \theta}\right) .
\end{gathered}
$$

As in the procedure for the pin, setting all $A_{k}$ to zero except one gives equations for determining $a_{k}$ and $b_{k}$ and nence the response of the plate to traction $N=i T=e^{i k \theta}$ on the hole. However, with the plate the procedure is sliyhtly different. No matter which $A_{k}$ is being considered, eq. 69 always yields an equation for $a_{0}$ and $b_{0}$, in addition to other equations involving other $a^{\prime} s$ and b's. This equation is

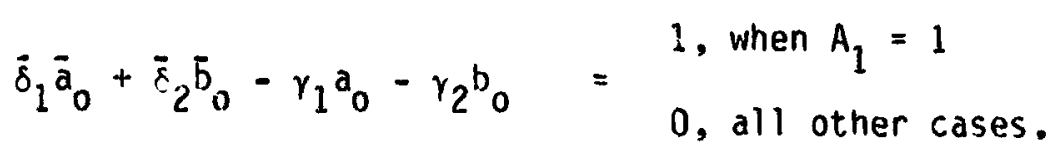

More information is needed to determine $a_{0}$ and $b_{0}$. The information can be obtained by exarnining the displacements. 
Because of the logrithmic term, there is the issue of single valvedness of the displacements. Using eq. 65 in eq. 61 and evaluating eq. 61 at $\theta=0$ and $\theta=2 \pi$, the following equation results:

$$
\left.\left(u_{r}+i u_{\theta}\right)\right|_{\theta=2 \pi}=\left(p_{1} a_{0}+p_{2} b_{0}-\bar{q}_{1} \bar{a}_{0}-\bar{q}_{2} \bar{b}_{0}\right) 2 \pi i+\left.\left(u_{r}+i u_{\theta}\right)\right|_{0=0}
$$

For single valuedness, the expression in parenthesis must be zero, i.e.

$$
p_{1} a_{1}+p_{2} b_{0}-\bar{q}_{1} \bar{a}_{0}-\bar{q}_{2} \bar{b}_{0}=0 \text {. }
$$

Equations 70 and 72 ;rovide the necessary equations to determine $a_{0}$ and $b_{0}$. Except for $k=1, a_{0}$ and $b_{0}$ are zero. For the case $k=1$, eqs. 70 and 72 were solved for numerically. Table 2 lists the coefficients $a_{k}$ and $b_{k}$ for various values of $k$. With Table 2 the solution to the plate, namely the responses $\left(u_{r}\right)_{k_{p l a t e}}$ and $\left(u_{\theta}\right)_{k_{p l a t e}}$ are available for use ir: eq. 28 .

\section{Details of the Numerical Scheme}

The collocation procedure included the points $\theta=0$ and $\theta=\pi$. Because of symmetry, the elasticity solutions for the pin and the plate due to traction $N-i T=e^{i k \theta}$ automatically yielded

and

$$
\left(u_{\theta}\right)_{k_{p i n}}=\left(u_{0}\right)_{k_{p l a t e}}=00 \theta=0, k=0, \pm 1, \ldots, \pm N .
$$

$$
\left(\tau_{r \theta}\right)_{k_{p \text { in }}}=\left(\tau_{r \theta}\right)_{k_{p l a t e}}=0 \Leftrightarrow \theta=\pi, k=0, \pm l, \ldots, \pm N .
$$


Table?

Coefficients of the stress functions $\phi_{1}$ and $\phi_{2}$ for the plate

nonzero

$A_{k}$
$A_{0}=1$
$a_{1}=\frac{\varepsilon_{2}}{\delta_{2} r_{1}-\delta_{1} r_{2}}$
$b_{1}=\frac{\delta_{1}}{\delta_{1} \gamma_{2}-\delta_{2} \gamma_{1}}$
$A_{-1}=1$
$a_{2}=\frac{\delta_{2}}{2\left(\delta_{2} \gamma_{1}-\delta_{1} \gamma_{2}\right)}$
$b_{2}=\frac{\varepsilon_{1}}{2\left(\delta_{1} \gamma_{2}-\delta_{2} \gamma_{1}\right)}$

$A_{+1}=1$

soln to eq. 70 and 72

$A_{-2}=1$

$a_{3}=\frac{\delta_{2}}{3\left(\delta_{2} \gamma_{1}-\delta_{1} \gamma_{2}\right)}$

$b_{3}=\frac{\delta_{1}}{3\left(\delta_{1} \gamma_{2}-\delta_{2} \gamma_{1}\right)}$

$A_{+2}=1$

$a_{1}=\frac{r_{2}}{\delta_{3} r_{1}-\delta_{1} r_{2}}$

$b_{1}=\frac{r_{1}}{\delta_{1} \gamma_{2}-\delta_{2} \gamma_{1}}$

$A_{-k}=1$

$a_{k+1}=\frac{\delta_{2}}{(k+1)\left(\delta_{2} \gamma_{1}-\delta_{1} \gamma_{2}\right)}$

$b_{k+1}=\frac{\delta_{1}}{(k+1)\left(\delta_{1} \gamma_{2}-\delta_{2} \gamma_{1}\right)}$

$A_{+k}=1$ $b_{k-1}=\frac{\gamma_{1}}{(k-1)\left(\delta_{1} \gamma_{2}-\delta_{2} \gamma_{1}\right)}$ 
These two conditions led to two columns and rows of eq. 28 being zero. The collocation procedure included points at $\theta=0$ and $\theta=\pi$ because there was one other condition at each point that had to be enforced, namely

$$
\left(u_{r}\right)_{\text {pin }}=\left(u_{r}\right)_{\text {plate }}-\delta \text { at } \theta=0
$$

and

$$
\sigma_{r}=0 \text { o } \theta=\pi
$$

Thus the two rows and columns representing eqs. 73 and 74 were el iminated from the set of linear equations, eq. 28. Failure to do this resulted in a singular snatrix. The number of equations and unknown $A_{k}$ 's solved for was reduced to $2 \mathrm{~N}-1$.

Values for $\alpha, \beta$, and $\delta$ were chosen. The system of $2 N-1$ equations was solved for the $A_{k}$ 's. In all likelinood the values of $\alpha$ and $\beta$ chosen did not satisfy all the interface and boundary conditions for that specific value of $\delta$. An iterative process then began to find values of $\alpha$ and $\beta$ that did. The key to the iteration process was the satisfaction of conditions which were a combination of a restatement of the conditions stipulated by eqs. 4-6 and statements regarding the physical realities of the problem. These conditions were:

at the hole edge $\begin{cases}\sigma_{r} \leqslant 0, \text { all } \theta & (77) a \\ \sigma_{r}=\tau_{r \theta}=0, \beta<\theta<\pi & (77) b \\ \sigma_{r}=\tau_{r \theta}=0, \theta=\beta & (77) c \\ \left|\tau_{r \theta}\right|<\mu\left|\sigma_{r}\right|, 0 \leqslant \theta<\alpha & (77) \mathrm{d} \\ \left|\tau_{r \theta}\right|=\mu\left|\sigma_{r}\right|, \theta=\alpha & \text { (77)e }\end{cases}$ 
Equation 77a states that the radial tractions had to be either compressive or zero, everywhere. Equation $77 \mathrm{~b}$ states the tractions had to be zero in the no-contact 2one. Equation $77 \mathrm{c}$ states that at the end of the contact zone the tractions had to be zero. Equation 77d states that the friction-induced shear traction had to be less than the Coulomb limit in the no-slip zone. Equation 77e states that at the end of the no-slip 2one, the friction induced shear stress was exactly the coulonb limit. Equations 77c and 77e are written explicitly : ocause behavior of $\sigma_{r}$ at $\theta=\beta$ and $\tau_{r \theta}$ at $\theta=\alpha$ were the most powerful indicators of convergence. To obtain a solution, the value of $\alpha$ was set to a value corresponding to the first collocation point away from $\theta=0$. Attention was focused on $\beta$ by selecting a reailistic value. Depending on whether the value of $\beta$ chosen was larger or smaller than the correct value, the $\sigma_{r}$ vs. $\theta$ relation had very distinctive characteristics near $\theta=\beta$. Fiyure 4 illustrates the nature of the solution for three values of $\beta$. The correct value of $\beta$ is denoted $\beta^{\star}$. If the value of $\beta$ chosen was larger than $\beta^{*}$, say $\beta=\beta_{1}, \sigma_{p}$ was tensile near $\theta=\beta_{1}$. If the value of $\beta$ chosen was smaller than $\beta^{*}$, say $\beta=\beta_{2}$, then $\sigma_{r}$ was not zero at $\theta=\beta_{2}$. By recognizing what the character of the $\sigma_{r}$ vs. $\theta$ meant relative to the assumed value of $\beta$, within 3 or 4 iterations a value of $\beta$ could be obtained which satisfied eqs. 77a-c. Iteration for a then began. This iteration procedure was similar in that the $\tau_{r \theta}$ vs. $\theta$ relation showed distinct characteristics near $\theta=\alpha$. These characteristics depended on whether the value of $a$ chosen was laryer or smaller than the co. ect $\alpha$. The characteristics near $\theta=\alpha$ are shown in fig. 5. The correct value of $\alpha$ is denoted as $\alpha^{*}$. If the value of $\alpha$ chosen was larger than $\alpha^{*}$, say $\alpha_{1}$, then at $\theta=\alpha_{1},\left|\tau_{r \theta}\right|$ exceeded $\mu\left|\sigma_{r}\right|$. If the value of $\alpha$ 
chosen was smaller than $\alpha^{*}$, say $\alpha=\alpha_{2}$, then at $\theta=\alpha_{2}, \mid \tau_{r \theta} i$ was less than $\mu\left|\sigma_{p}\right|$. Within 3 or 4 iterations a solution for $\alpha$ was obtained which satisfied eqs. 77d and $c$. The value of $\beta$ was then rechecked and adjusted if necessary. Generally there was little coupling between the two variables.

For smaller values of $\mu\left(e_{. g} \mu=0.2\right)$ the no-slip region was found to be small. Often the no-slip region was smaller than the spacing between the collocation point at $\theta=0$ and the point next to it. In these cases the no-slip condition was satisfied only at $\theta=0$. Since by the symmetric nature of the solution there was no relative tangential motion at $\theta=0$ anyway, the no-slip region could only be determined to be less than the collocation point spacing.

Overall, the convergence of the solution was found to be dependent on the location and number of collocation points used to satisfy the boundary conditions. It was important to have the collocation points close to being equally spaced around the hole. Forty collocation points around the half-circle were used to obtain the numerical results presented in the next section. Thus the results to be presented used 78 unknown $A_{k}{ }^{\prime} s$ in the series representing $\mathrm{N}-\mathrm{iT}$. The 78 terms were divided evenly between negative $k$ and positive $k$. Twenty to 30 collocation points gave reasonable results when compared to similar cases from previous investigations. Forty points represented the upper limit of the interactive computer used in the analysis. Fewer than 20 points resulted in pour agreement with established work. 


\section{Numerical Results}

Mumerical results were obtained in order to assess the effects of the various parameters on the stresses around the hole. The numerical study considered plates with varying degrees of orthotropy. The results for four different plates are presented here. In the context of fiberreinforced composite plates, these four plates represent four different laminates. The material properties of each plate ire indicated in Table 3, as are the laminates they represent. The plate properties considered coincide with the basic properties used by Crews et al. [10] and represent a graphite-epoxy material.

Plate $A$ represents an extreme in degree of orthotropy. The piate is much stiffer in the load direction than it is perpendicular to the load direction. This represents a graphite-epoxy plate made with all the fibers in the direction of the load. Plate $B$ represents the other extreme, being much softer in the load direction than perpendicular to the load direction. Plate B represents a graphite-epoxy plate with all the fibers perpendicular to the load direction. Plate $C$ represents a plate with properties representative of laminates which have the same inplane stiffness in all directions. Such laminates are referred to as quasi-isotropic laminates. Plate D represents a moderately orthotropic laminate, being three times stiffer in the load direction than it is perpendicular to the load. These four plates will be used to illustrate the effect of pin flexibility and the plate's elastic properties on the stresses around the hole. Plate $D$ then will be used to illustrate the effect of friction, clearance, and pin displacement on stresses. 
Table 3

\section{Material Properties of Plates}

$\begin{array}{cccccc}\text { Plate } & E_{x} & E_{y} & G_{x y} & v_{x y} & \text { representative } \\ & \text { Msi } & \text { Msi } & \text { Msi } & & \text { laminate* } \\ \text { A } & 21.3 & 1.58 & 0.930 & 0.38 & 0^{\circ} \\ \text { B } & 1.58 & 21.3 & 0.930 & 0.028 & 90^{\circ} \\ \text { C } & 8.40 & 8.40 & 3.20 & 0.310 & \left(0^{\circ} / \pm 45^{\circ} / 50^{\circ}\right)_{s} \\ \text { D } & 12.4 & 3.73 & 3.21 & 0.667 & \left(0^{\circ}{ }_{2} / 45^{\circ}\right)_{5}\end{array}$

*fiber angles relative to $+x$ direction

Figures 6-9 show the effects of pin flexibility and plate material properties on the stresses around the hole. The three pin flexibilities used were: a rigid pin, a steel pin $\left(E=30 \times 10^{6} \mathrm{psi}\right)$, and an aluminum pin $\left(E=10 \times 10^{6}\right.$ psi). Poisson's ratio of the pin had very little effect on the numerical results and a value of 0.3 was used. In each of figs. 6-9 the coeffirient of friction between the plate and the pin was assumed to be 0.2 . This represents a reasonable value for metal on yraphite-epoxy. The non-dimensional clearance between the pin and plate, $i / R$, was 0.01 and the nondimensional pin displacement, $\delta / R$, was 0.035. Realistic values of pin/hole clearance vary from application to application but $\lambda / R=0.01$ is representative. Each figure has the same scale, for easy comparison, and each figure illustrates the three stresses at the hole edge, i.e. the radial stress $\sigma_{r}$, the circumferential

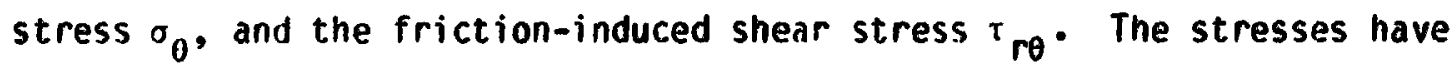


been nondimensionalized by the bearing stress and the behavior of the stresses with circumferential location is illustrated. The bearing stress is defined as the pin load, $P$, divided by the product of plate thickness and hole diameter. Also shown in figs. 6-9 is the cosinusoidal radial stress distribution often assumed. Thus the figures illustrate the two assumed pin/hole interaction models used in previous investigations, i.e. rigid and cosinusoidal, as well as the current more realistic one. As a check on the numerical solution, the stresses $\sigma_{r}$ and $\tau_{r} \theta$ were integrated around the hole edge to determine the total load acting on the hole. The integration was always within $0.01 \%$ of $P$. When examining figs. 6-9, three conclusions are obvious. First, it is clear pin flexibility is not a big factor in determining the stresses at the hole edge. Until now, no investigation has shown this explicit1y. Second, the degree of orthotropy strongly influences the peak stresses and the distribution of the stresses around the nole. The highly orthotropic plate $A$ in fig. 6 has a stress concentration factor of 2 for the circumferential stress compared to the 1.2 of the quasiisotropic plate $C$ in fig. 8. Third, the often-assumed cosinusoidal distribution is not generally accurate. It is a serious misrepresentation for plate $B$ and, due to pin/ hole clearance effects, does not properly represent the contact region in any situation. It will be seen that the character of $\sigma_{r}$ near $\theta=0$ is determined by friction while the character of ${ }^{\circ} r$ near $90^{\circ}$ is determined by pin/hole clearance. Thus for particular friction and clearance levels, the cosinusoidal distribution could be a good representation. However, in all cases, the rigid pin assumption is better. In all the cases shown in figs. 6-8, the circumferential stress $\sigma_{0}$ is negative at $\theta=0$. This is somewhat counter to intuition 
but the result, as will be seen shortly, is due to frictional effects. This was found in Wilkinson's [5] analysis and it has been measured [23]. The decrease in the magnitude of $\sigma_{r}$ as $\theta \rightarrow 0$, in plate $A$ for example, is also due to friction.

Table 4 irdicates the contact and no-slip angle determined by the iterative procedure for figs. 6-9 and for figures to be discussed. The location of the maximum hoop stress is also indicated in the table. The location of the maximum hoop stress, while generally occurring near the end of the contact region, depends on the plate's elastic properties. A plate representing a laminate with fibers at $445^{\circ}$ and $-45^{\circ}$ relative to the load direction experiences a maximum hoop stress at roughly $\theta=45^{\circ}$.

Figure 10 illustrates the effect of friction on the stresses at the nole edge of plate $D$. Three values of friction were examined for the case of a steel pin with clearance $\lambda / R=0.01$ and displacement $\delta / R=$ 0.035 . The values of friction examined were: $\mu=0,0.2$, and 0.4 . The 1st and 3rd values of $\mu$ represent extremes and were chosell to bracket the effects of friction. The most significant effect of friction, besides influencing the level of shear stress, is its influence on peak stresses. Increasing friction tends to decrease the maximum radial stress at $\theta=0$. This effect occurred for all situations studied. Friction also increased the maximum circumferential stress near the end of the contact region. Friction had some effect on the actual contact 2one. Because of the effect of friction at $\theta=0$, the cosinusoidal representation (not shown) is closer to the frictionless case than the other cases. In fact, with no friction and no clearance, the cosinusoidal assumption is close for this particular plate. 


\section{Table 4}

Location of Maximum Hoop Stress, Contact and

No-Slip Regions for Various Cases

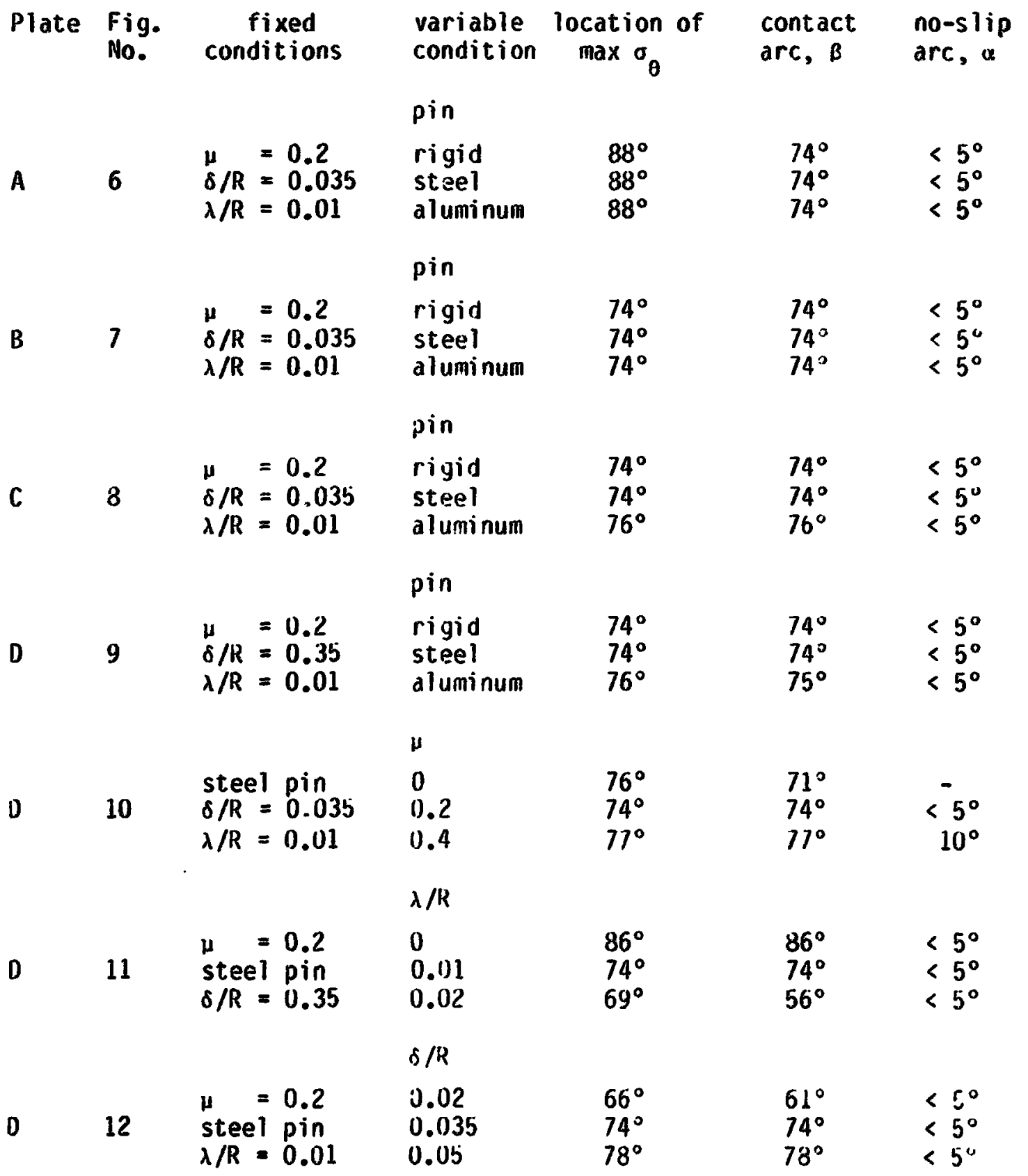


Figure 11 illustrates the effect of pin/hole clearance on the holeedge stresses in plate $D$. The three levels of clearance illustrated are $\lambda / R=0.0,0.01,0.02$. The value $\lambda / R=0$ is of ten referred to as the snug-fit, push-fit, or perfect-fit pin. The value of $\mu$ used was 0.2 and $\delta / R$ was 0.035 . The $p$ in was steel. The most effect $p i n / h o l e c l e a r-$ ance has is in the location of the peak circumferential stress. Increasing the clearance moves the peak stress location toward $\theta=0$. This is a direct consequence of the rapid decrease in contact zone with increasing clearance. Increasing the clearance also causes more of a region to experience high circumferential stress. Table 4 quantifies the effect of clearance on contact angle. As expected, the value of the peak radial stress is sensitive to the level of clearance. With less contact area the pin naturally loads the contact region more.

Finally fig. 12 shows the effect of increasing $p$ in displacement on the stresses. The pin is steel, the coefficient of friction is 0.2 , and the clearance is $\mathbf{0 . 0 1}$. Three values of pin displacement are considered: $\delta / R=0.02,0.035$ and 0.05 . The most significant effects are with the contact arc and the location of the peak circumferential stress. With increasing pin displacement the contact arc increases and the location of the peak circumferential stress moves toward $\theta=90^{\circ}$. Obviously increasing pin displacement increases the maynitude of the actual stresses. However, in the nondimensional sense shown in fig. 12, the stress inagnitudes are not strongly influenced. The peak nondimenstonal radial stress actually decreases some with increasing pin displacement due to the longer contact arc. 


\section{Additional Comments}

Two other issues should be addressed before closing. The first issue deals with the functional behavior of the stresses between collocation points. The second issue deals with friction.

Figures 6-12 were drawn by hand-fairing lines through the numerical values of the stresses computed at the collocation points. When a Fourier series representation of a function is used in conjunction with collocation, there is the issue of behavior of the series representation between the collocation points. In addition, here there is the issue of che behavior near the end of the contact zone, where the stress state is rapidly changing with $\theta$. When the numerical values of $A_{k}$ were substituted into eq. 7 and $N$, for example was computed as a function of $\theta$, the function did indeed oscillate between collocation points. For a typical situation, for $\theta<\beta$ the amplitude of the oscillation was less than $2 \%$ of its maximum value. Just beyond $\theta=\beta$ the amplitude of the oscillation increased, but not markedly. Oscillation to a certain degree was expected. Wilson and Goree [24] discussed a dual-series approach to a contact problem which resulted in very little oscillation anywhere. Such an approach could have been used in this problem. However, nothing other than minimal oscillations were experienced with the rumerical approach here and so the issue of other numerical schemes did not arise. Concerning friction, Dundurs and Comninou [25] have shown that at the transition point between no-slip and slip, the slope of $\tau_{r \theta}$, with respect to $\theta$, becomes infinite. With the approach here such behavior would never be realized and thus the results are in error in that regard. An alternative approach would have been to build in a special friction-induced shear function which did indeed yield an infinite slope 
of the transition point. However, it is felt that the results presented are not in considerable error for not accurately representing this effect.

\section{Concluding Remarks}

This paper has used elasticity solutions and a numerical procedure to study the stress distributions around a hole in a pin-loaded orthotropic plate. In particular, the effects of pin elasticity, friction and clearance have been studied. In addition, the effects of the plate's elastic properties on the stress distribution has been assessed. It can be concluded that, within the context of this study, pin elasticity is not an important variable. Pin/hole clearance is an important variable and friction does effect the stress distributions. Further studies are warranted in the area of finite-geometry plates, a circumferentially variable coefficient of friction, or alternatively, a nore general (non-Coulomb) friction model. Perhaps experimental investigations into the slip and no-slip zones are necessary before more general friction laws are incorporated.

Acknowledgements

The work reported on here was supported by the NASA-Virginia Tech Composites Program, Cooperative Agreement NCCI-15 with the NASA Langley Research Center. 


\section{References}

1. J. P. Waszczak, T. A. Cruse, "Failure Mode and Strength Predictions of Anisotropic Bolt Bearing Specimens," J. Comp. Mat., Vol. 5, July 1971, pp. 421-425.

2. D. W. Oplinger W. and K. R. Ganuthi, "Stresses in Mechanically Fastened Orthotropic Laminates," 2nd Conf. of Fib. Comp. in Flight Vehicle Design, May 1974, pp. 813-841.

3. D. W. Oplinger, K. R. Ganani, "Analytical Studies of Structural Performance in Mechanically Fastened Fiber-Reinforced Plates," Army Symp. on Solid Mach., Sept. 1974, pp. 211-240.

4. T. de Jong, "Stresses Around Pin-Loaded Holes in Elastically Orthotropic or Isotropic Plates," J. Comp. Mat., Vol. 11, July 1977, pp. 313-331,

5. T. L. Wilkinson, "Stresses in the Neighborhood of Loaded Holes in Wood with Applications to Bolted Joints," Ph.D. Dissertation, Iniv. of Wisc.-Madison, Available through Univ. M"crofilms, 1978.

6. B. L. Agarwal, "Static Strength Prediction of Bolted Joint in Composite Materials," AIAA Journal, Vol. 18 No. 11, Nov. 1980, pp. 1371-1375.

7. P. D. Mangalgiri, B. Dattaguru, "Elastic Ana lysis of Pin Joints in Composite Plate," Rep. No. ARDB-STR-5014, Dept. Aeronaut. Engr., Ind. Inst. Sci., Nov. 1980.

8. C. M. S. Wong, F. L. Matthews, "A Finite Element Analysis of Single and Two-Hole Bolted Joints in Fibre-Reinforced Plastic," J. Comp. Mat., Vol. 15, Sept. 1981, pp. 481-491.

9. T. de Jong, H. A. Vuil, "Stresses Around Pin-Lo.3ded Holes in Elastically Orthotropic Plates with Arbitrary Load li rection," Report LR-333, Dept. of Aerospace Engr., Delft Univ. Tech., Sept. 1981.

1.. J. H. Crews, C. S. Hong, "Stress-Concentration Factors for Finite 0:thotropic Laminates with a Pin-Loaded Hole," NASA TP 1862, May 1981.

11. S. P. Garbo, J. M. Ogonowski, "Effects of Variances and Manufacturing Tolerances on the Design Strength on Life of Mechanically Fastened Composite Joints," AFWAL-TR-81-3041, Vols. 1-3, Apri 11981.

12. Soni, S. R., "Failure Analys is of Composite Laminates with a Fastener Hole," ASTM STP 749, Joining of Composite Materials, 1981, p. 145-164. 
1... T. de Jong, "The Influence of Friction on the Theoretical Strength of Pin-Loaded Holes in Orthotropic Plates," Rep. No. LR-350, Dept. of ferospace Engr., Delft Univ. Tech., March 1982.

14. F. L. Matthews, C. M. Hong, S. Chryssafitis, "Stress Distribution Around a Single Bolt in Fibre-Reinforced Plastic," Composites, Vol. 13, No. 3, July 1982, pp. 316-322.

15. R. E. Rowlands, M. U. Rahman, T. L. Wilkinson, Y. I. Chiang, "Single- and Multiple-Bolted Joints in Orthotropic Materials," Composites, Vol. 13, No. 3, July 1982, pp. 273-278.

16. F. K. Chang, R. A. Scott, G. S. Springer, "Strength of Mechanically Fastened Composite Joints," J. Comp. Mat., Vol. 16, Mov. 1982, Pp. 470-494.

17. Bickley, W. G., "The Distribution of Stress Round a Circular Hole in a Plate," Phil. Trans. Royal Soc. (London), Vol. 227A, July 1928, P. $383-415$.

18. Nisida, M. and Saito, H., "Stress Distributions in a Semi-Infinite Plate Due to a Pin Determined by Interferometric Method," Experimental Mechanics, Vo1. 6, No. 5, May 1966, p. 273-279.

19. N. I. Muskhelishvili, Some Problems of the Mathematical Theory of Elasticity, Trans. J. R. M. Radok, Noordhoff, 1977.

20. P.ao, A. K., "Elastic Analysis of Pin Joints," Computers and Structures, Vol. 9, 1978, Pp. 125-144.

21. M. Milne-Thomson, Plane Elastic Systems, 2nd Ed., SpringerVerlag, 1968.

22. S. G. Lekhnitskii, Anisotropic Plates, Trans. S. W. Tsai and T. Cheron, Gordon \& Breach, 1968.

23. Hyer, M. W. and Liu, D., "Photoelasti: Determination of Stresses in Mult iple-pin Connectors," Experimental Mechanics, Vol. 23, No. 3, Sept. 1983, pp. 249-256.

24. Wilson, H. B., Jr. and Goree, J. G., "Axisymmetric Contact Slresses About a Smooth Elastic Sphere in an Infinite Solid-Stressed UniformIy at Infinity," J. Applied Mechanics, Vol. 34, No. 5, 1967, pp. $961-966$.

25. Dundurs, J. and Comninou, M., "An Educational Elasticity Problem with Friction, Part 1: Loading, and Unloading for Weak Friction, $J$. Applied Mechanics, Vol. 48, No. 4, 1981, Pp. 841-845. 


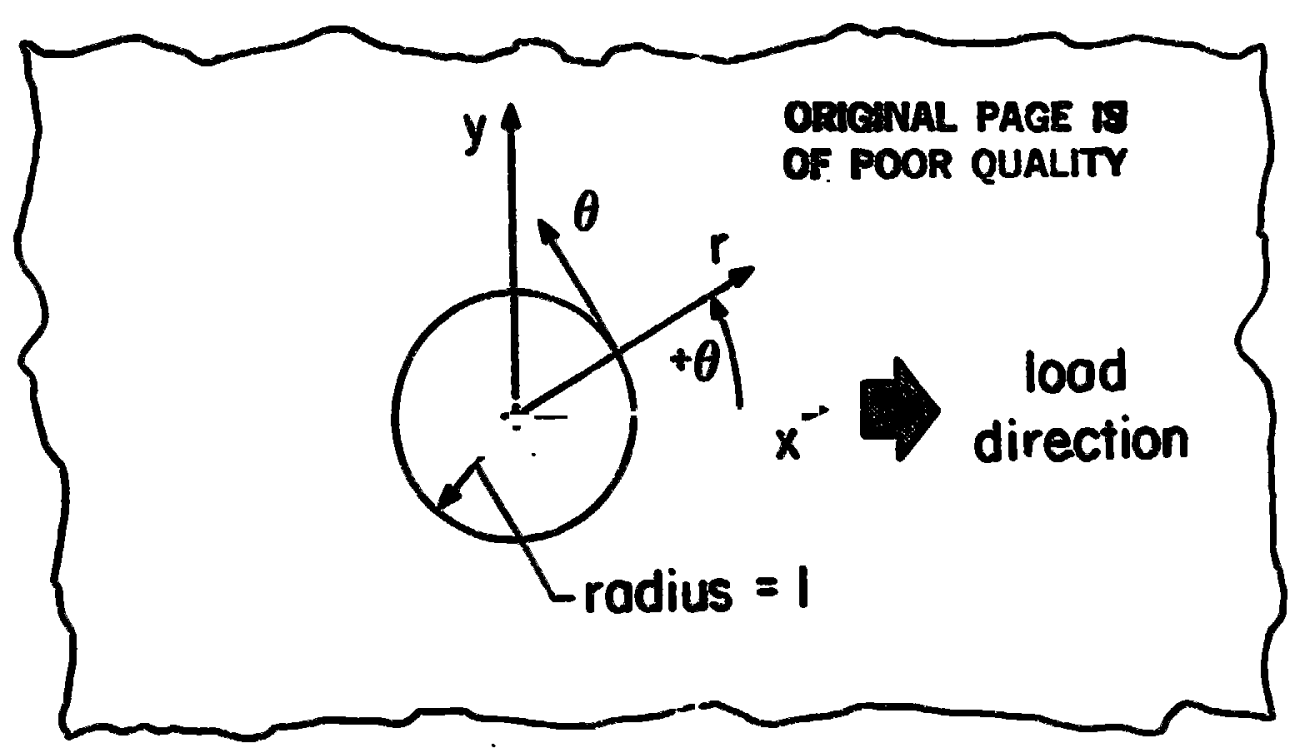

(a)

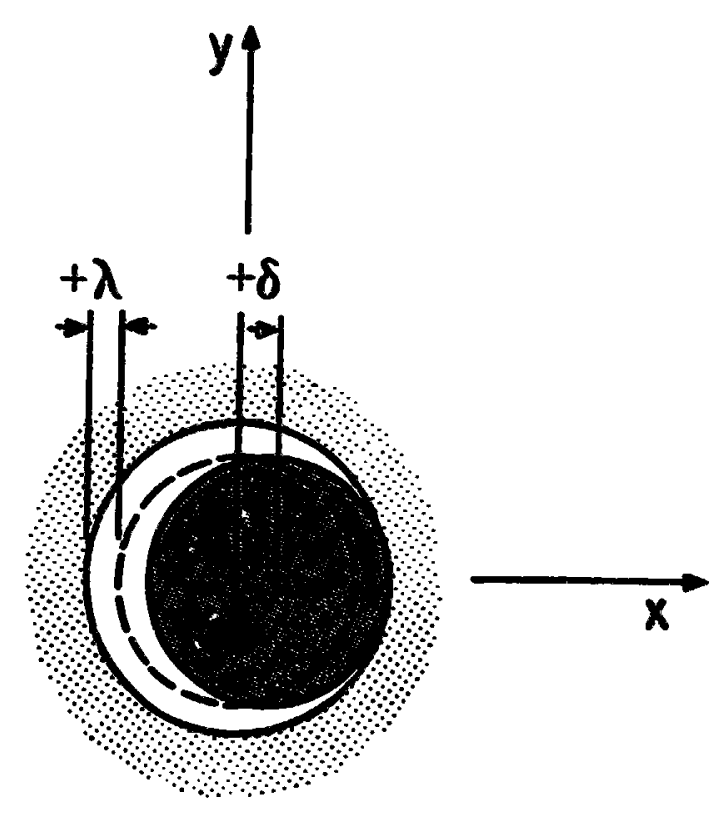

(b)

Fig. 1: a) Geometry and coordinate system used in analysis.

b) Definition of clearance, $\lambda$, and pin displacement, $\delta$. 


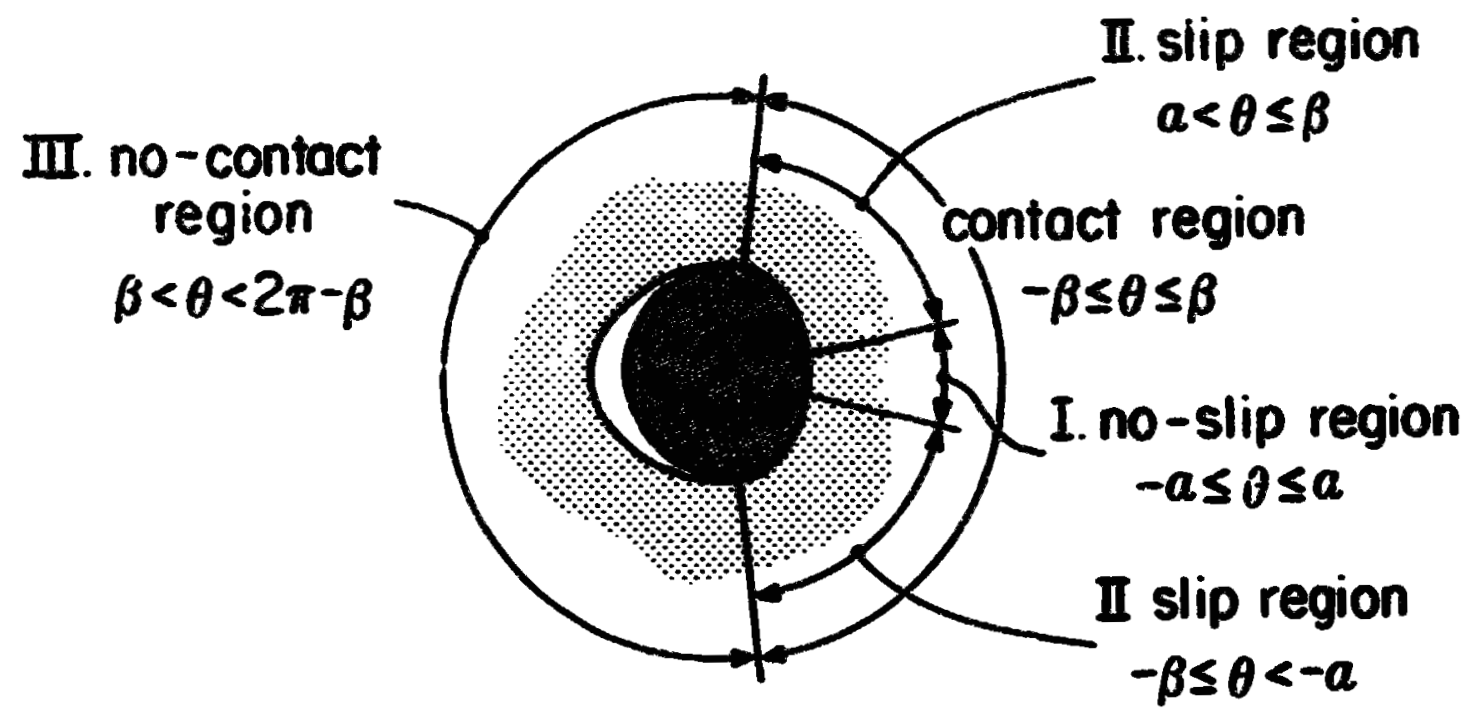

Fig. 2: Important regions at pin/hole boundary. 


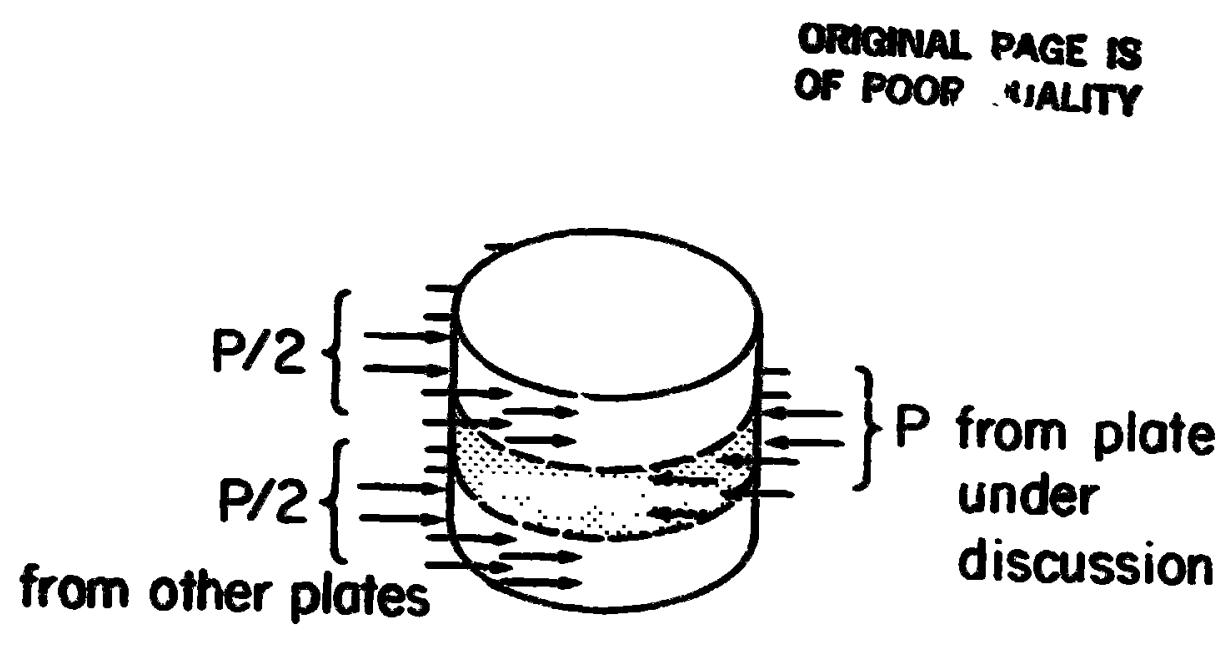

(a)

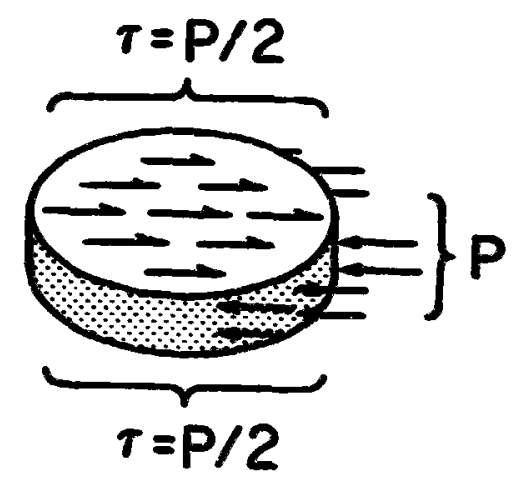

(b)

Fig. 3: Idealization of pin load: a) pin in double shear; b) section of $p$ in within plate thickness. 
ORIGNAL PSAE IY

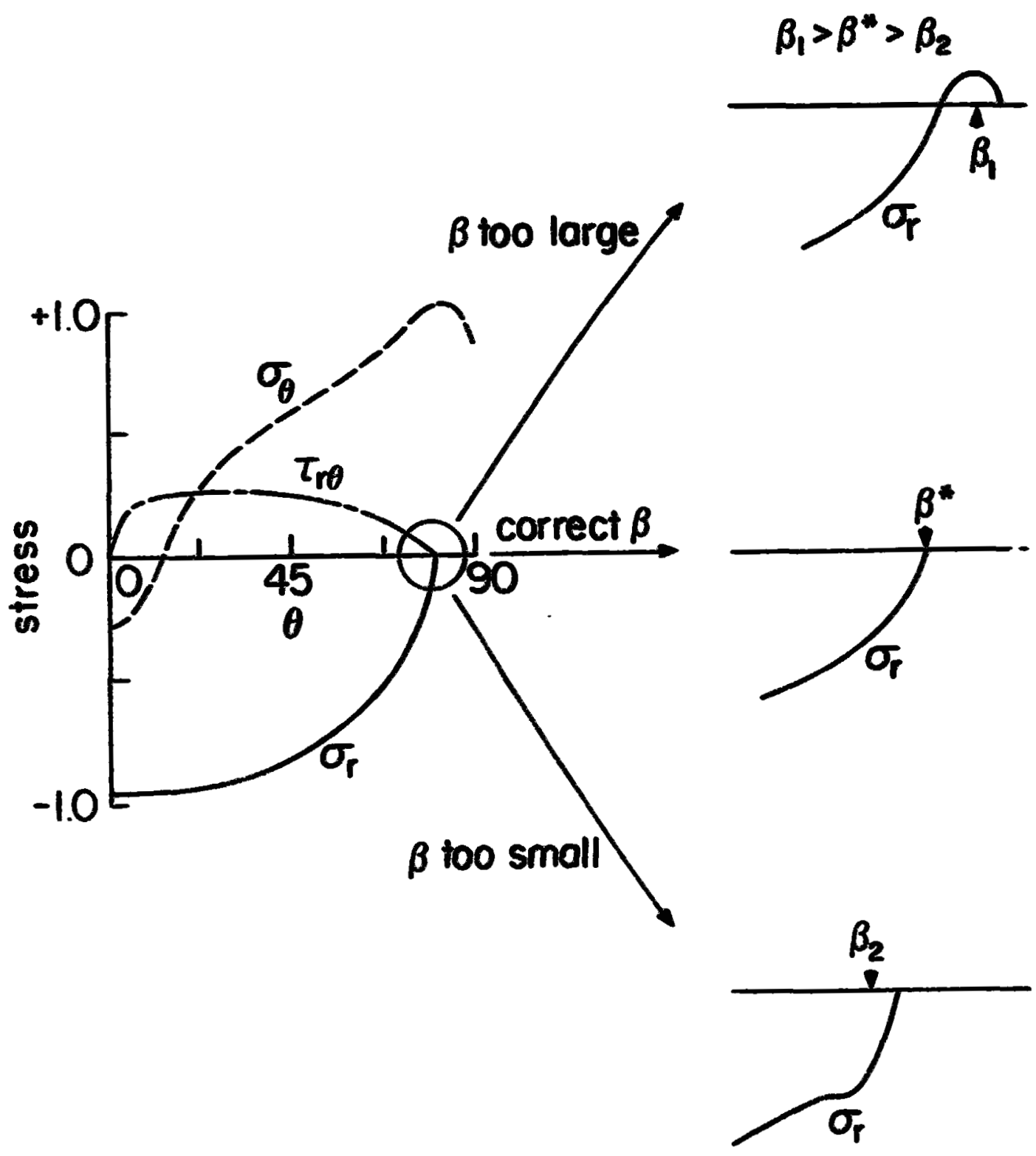

Fig. 4: Choosing the correct value of $\beta$. 


\section{omenal page is \\ OF POOR QUALITY}
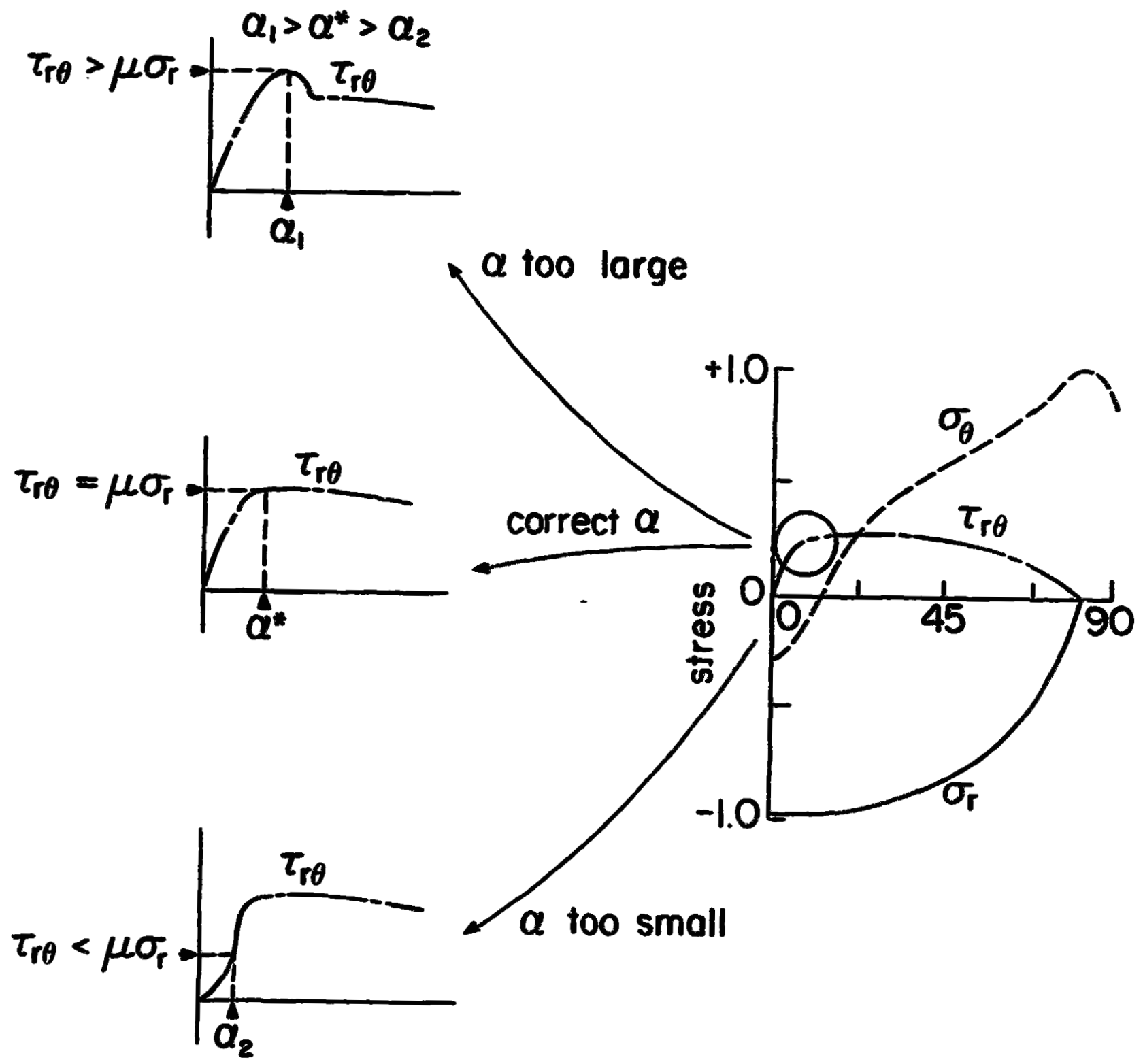

Fig. 5: Choosing the correct value of $\alpha$. 


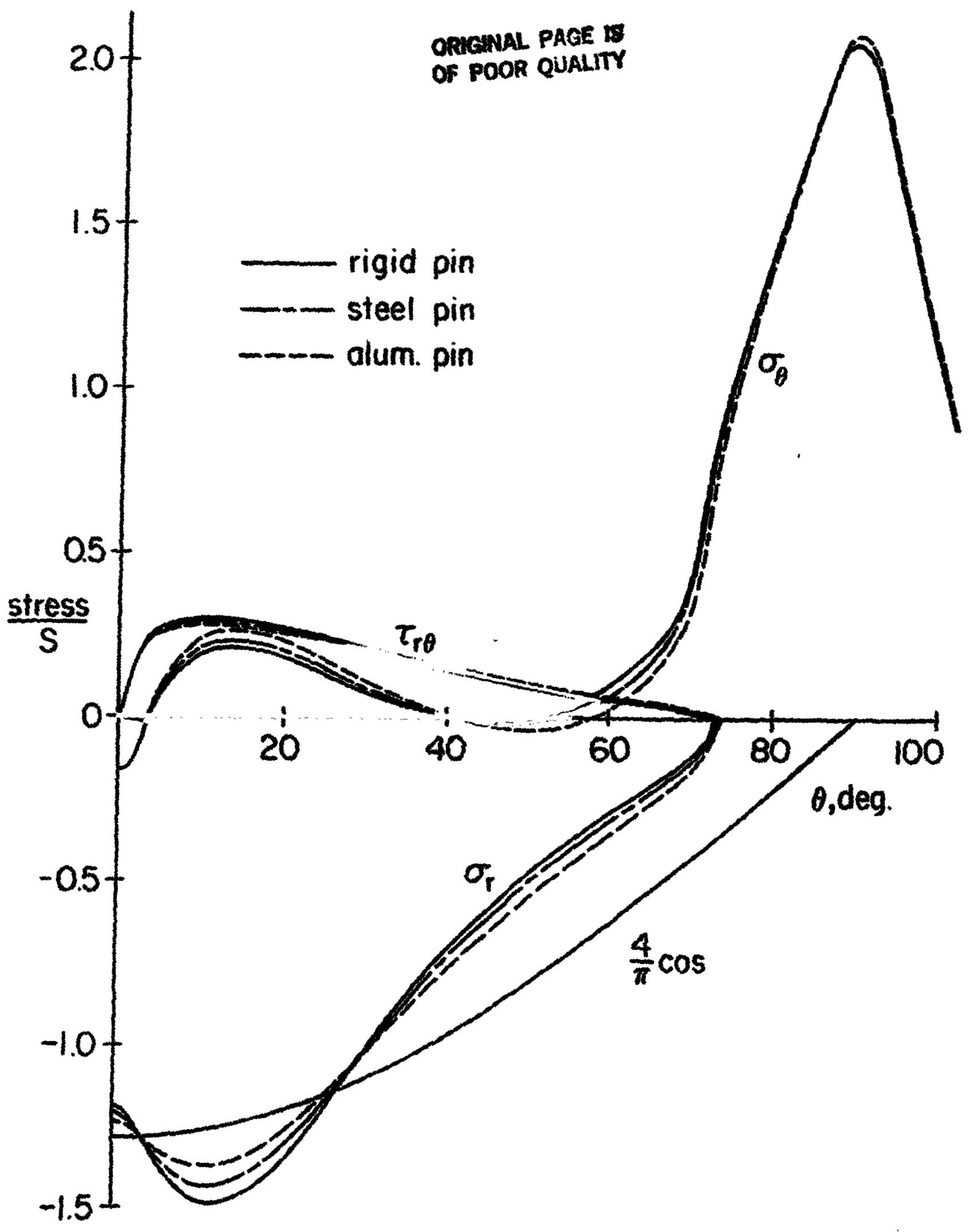

Fig. 6: Stresses around hole in plate $A$. 


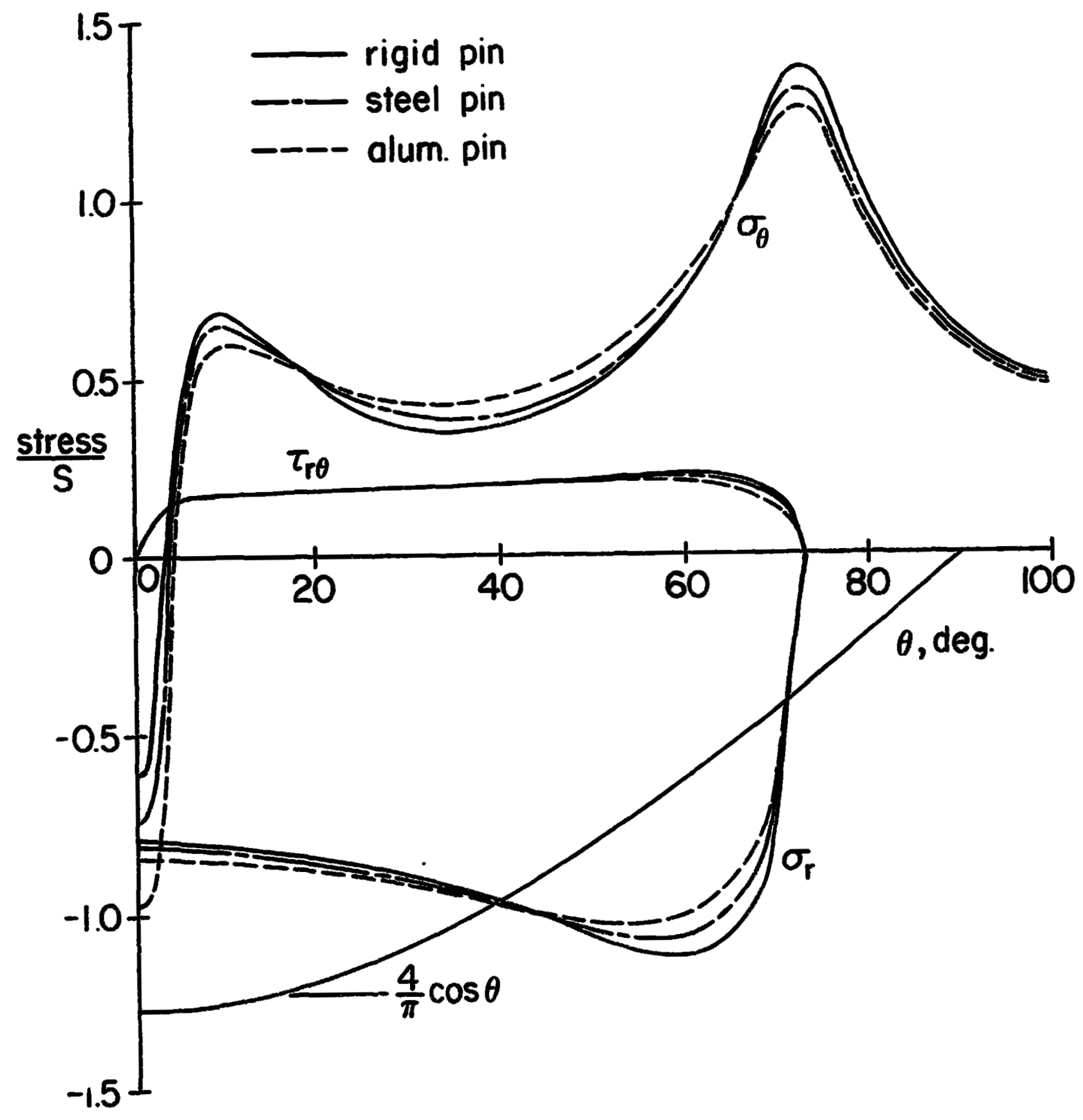

Fig. 7: Stresses around nole in plate $B$. 


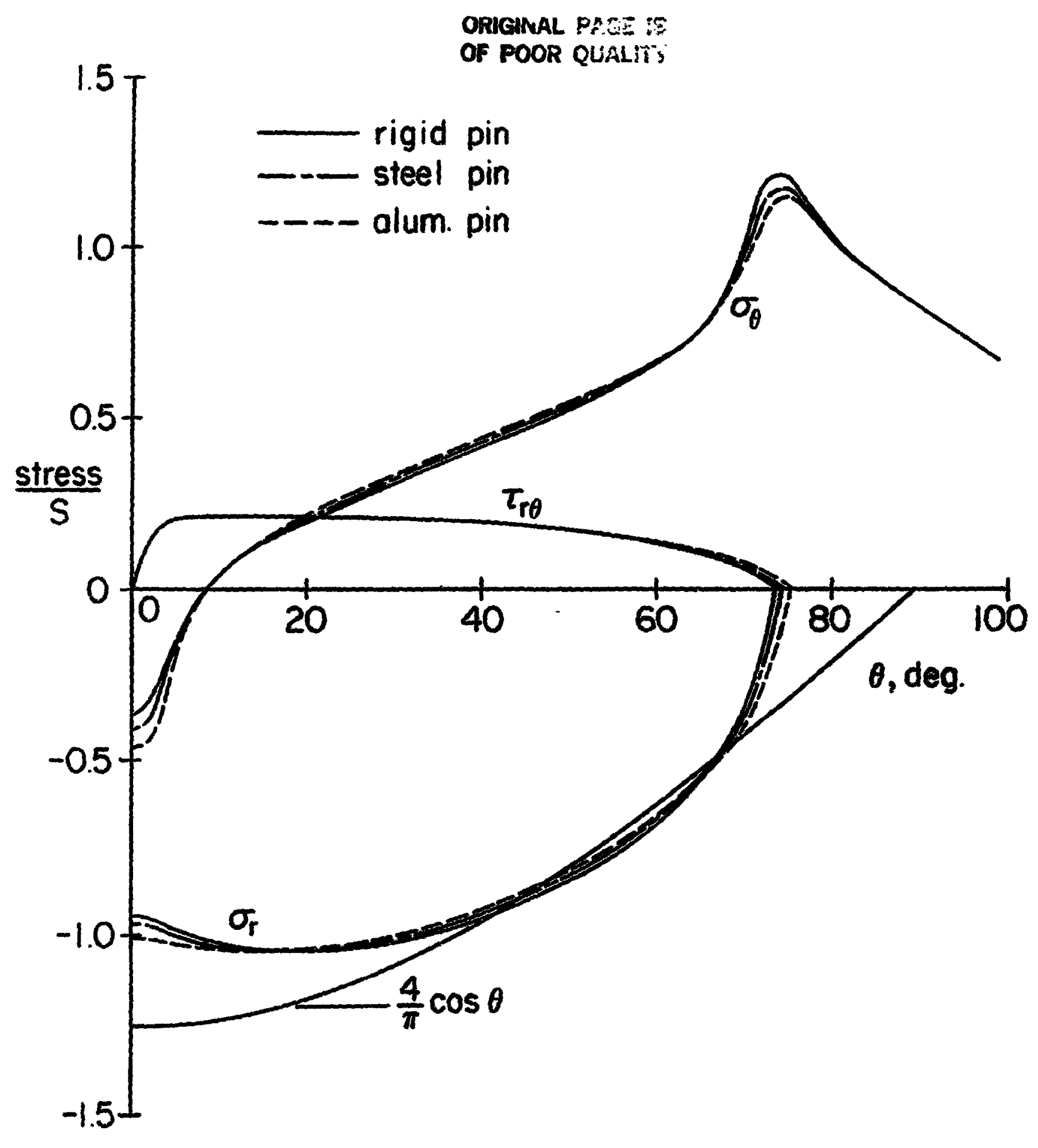

Fig. 8: Stresses around nole in plate $C$. 
ORIGINAL P:RE IJ

OF POOR QUALITY

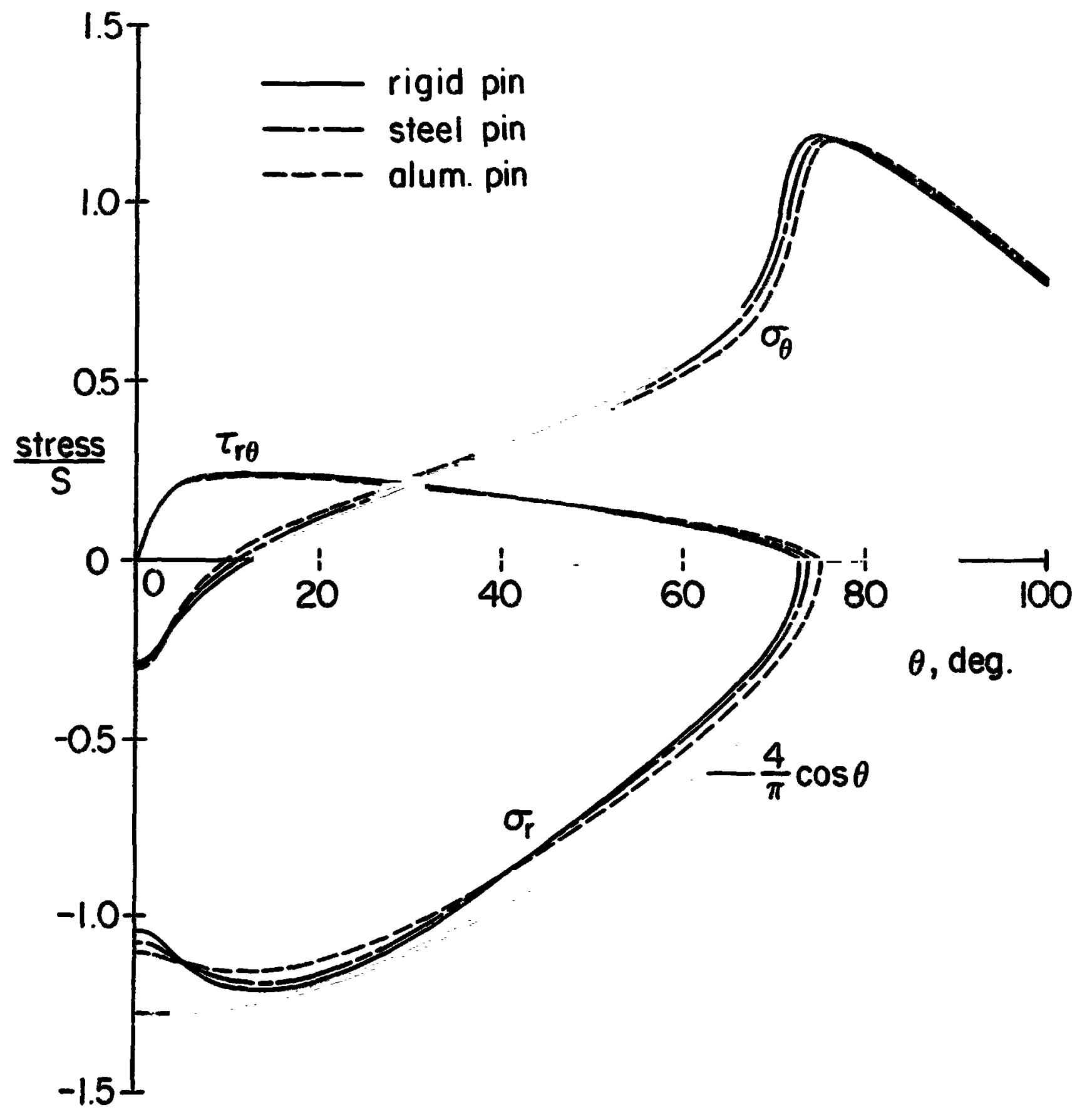

Fig. 9: Stresses around hole in plate D. 
ORIENAL PAEE IS

OF POOR QUALIT

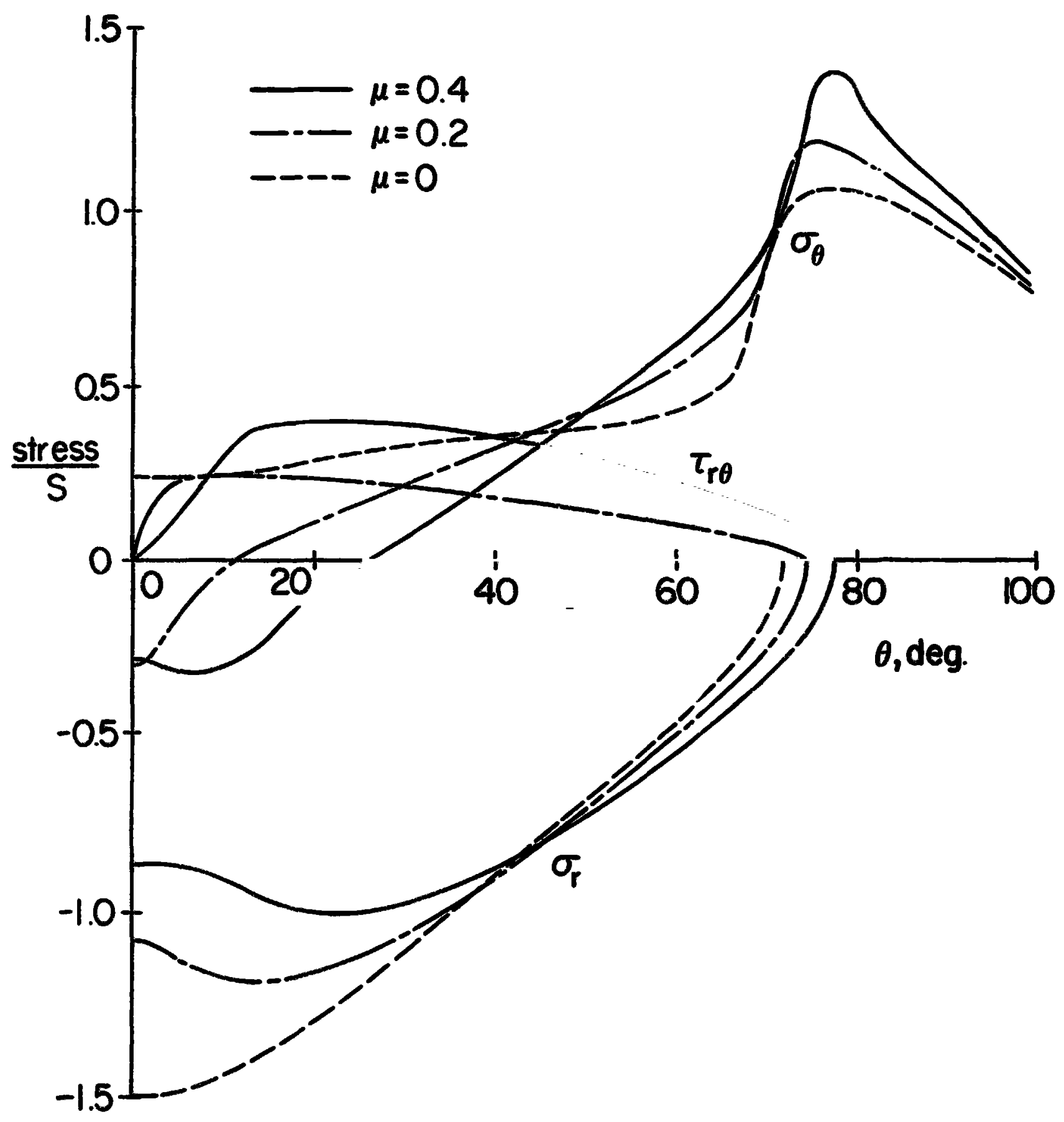

F1g. 10: Effect of friction on stresses. 


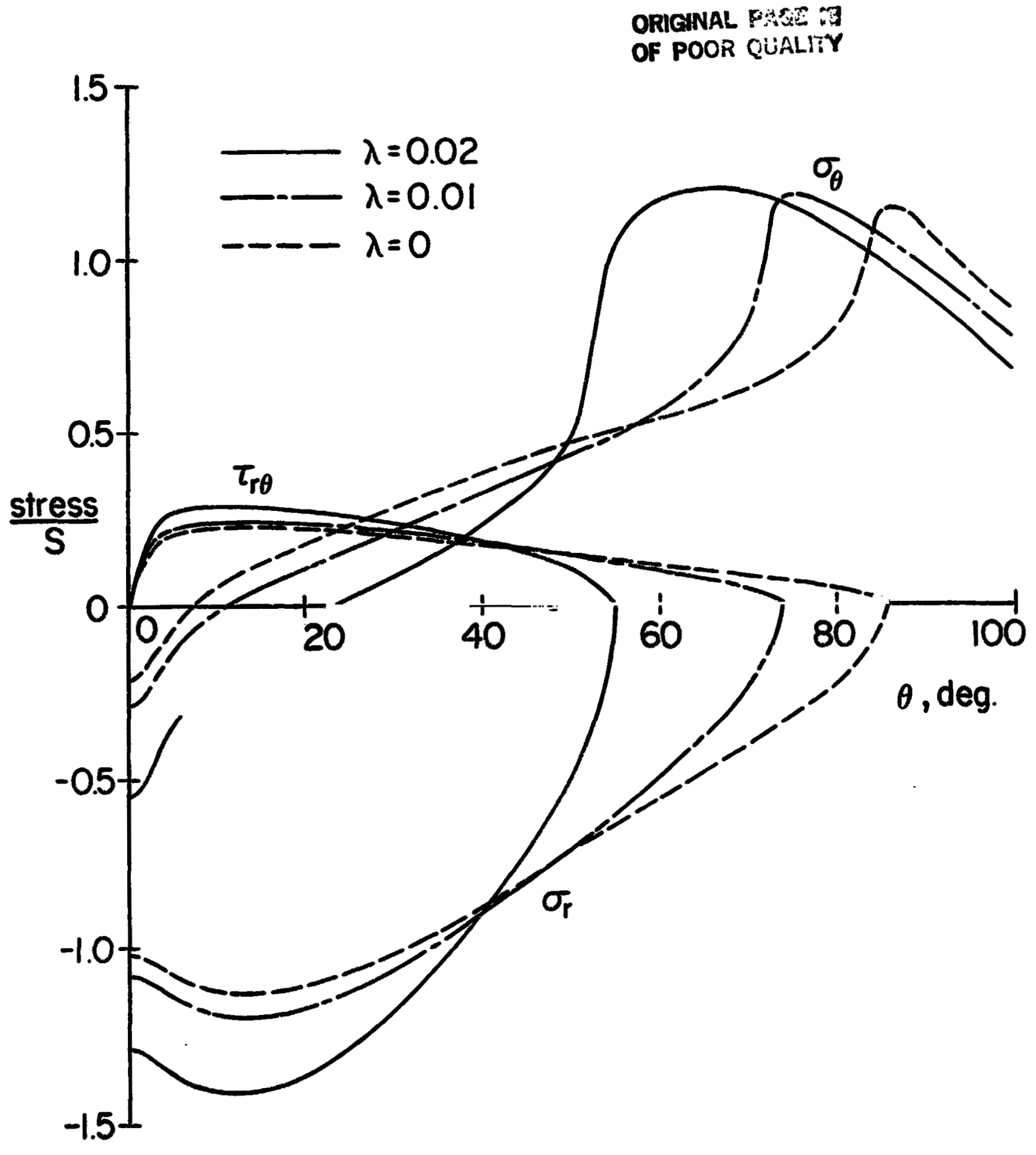

Fig. 11: Effect of clearance on stresses. 
ORIGINAL PAGE IS

OF POOR QUALITY

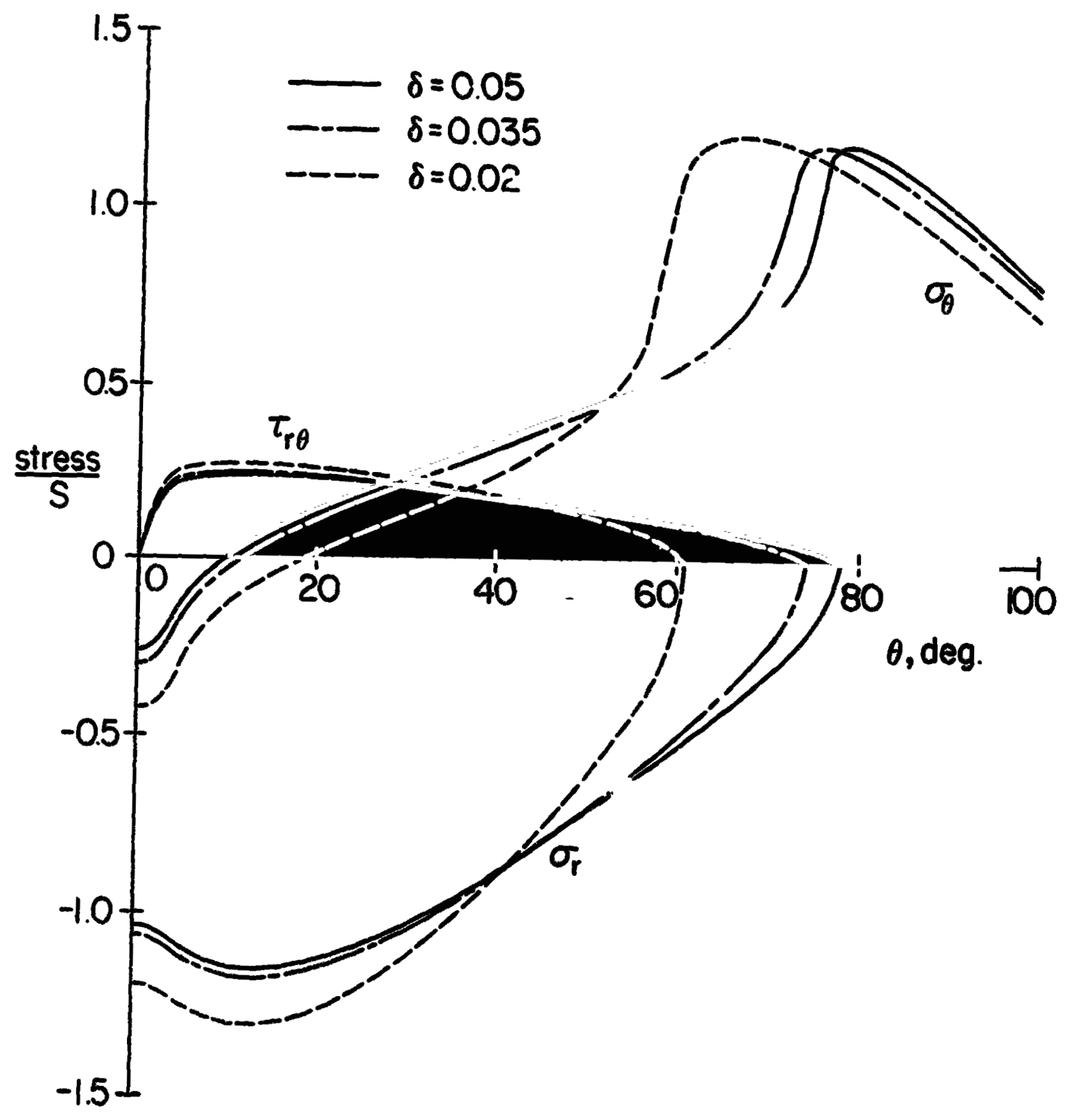

Fig. 12: Effect cf pin displacement on stresses. 